\title{
Seismic tests for solar models with tachocline mixing
}

\author{
A. S. Brun ${ }^{1,2}$, H. M. Antia ${ }^{3}$, S. M. Chitre ${ }^{4}$, and J.-P. Zahn ${ }^{2}$ \\ 1 JILA, University of Colorado, Boulder, CO 80309-0440, USA \\ 2 LUTH, Observatoire de Paris-Meudon, 92195 Meudon, France \\ e-mail: jean. paul-zahn@obspm.fr \\ 3 Tata Institute of Fundamental Research, Homi Bhabha road, Mumbai 400005, India \\ e-mail: antia@tifr.res.in \\ ${ }^{4}$ Department of Physics, University of Mumbai, Mumbai 400098, India \\ e-mail: kumarchitre@hotmail.com
}

Received 24 February 2002 / Accepted 31 May 2002

\begin{abstract}
We have computed accurate 1-D solar models including both a macroscopic mixing process in the solar tachocline as well as up-to-date microscopic physical ingredients. Using sound speed and density profiles inferred through primary inversion of the solar oscillation frequencies coupled with the equation of thermal equilibrium, we have extracted the temperature and hydrogen abundance profiles. These inferred quantities place strong constraints on our theoretical models in terms of the extent and strength of our macroscopic mixing, on the photospheric heavy elements abundance, on the nuclear reaction rates such as $S_{11}$ and $S_{34}$ and on the efficiency of the microscopic diffusion. We find a good overall agreement between the seismic Sun and our models if we introduce a macroscopic mixing in the tachocline and allow for variation within their uncertainties of the main physical ingredients. From our study we deduce that the solar hydrogen abundance at the solar age is $X_{\text {inv }}=0.732 \pm 0.001$ and that based on the ${ }^{9} \mathrm{Be}$ photospheric depletion, the maximum extent of mixing in the tachocline is $5 \%$ of the solar radius. The nuclear reaction rate for the fundamental $p p$ reaction is found to be $S_{11}(0)=4.06 \pm 0.0710^{-25} \mathrm{MeV}$ barns, i.e., $1.5 \%$ higher than the present theoretical determination. The predicted solar neutrino fluxes are discussed in the light of the new SNO/SuperKamiokande results.
\end{abstract}

Key words. Sun: abundances - Sun: interior - Sun: oscillations - neutrinos

\section{Introduction}

Over the past decade our understanding of the solar interior has improved significantly. Today with the precise helioseismic data available from the GONG (Global Oscillation Network Group) ground based instruments and the SOHO (SOlar and Heliospheric Observatory) space experiments (Gough et al. 1996; Thompson et al. 1996; Fröhlich et al. 1997; Gabriel et al. 1997; Schou et al. 1998), the detailed internal structure and complex dynamics of our star can be inferred with reasonable accuracy using inversion techniques. In addition to sound speed $c$ and density $\rho$ profiles, the internal rotation rate $\Omega$ can also be inferred. It reveals that on the top of an almost uniformly rotating radiation zone (with a rotation period of about 28 days), the bulk of the convection zone is differentially rotating with properties close to what is deduced from sunspot tracking, i.e., a period at the equator of 25 days and at the pole of 33 days corresponding to a contrast $\Delta \Omega$ of $30 \%$. The sharp transition region between these two distinct zones, located around $0.7 R_{\odot}$, has been called the tachocline

Send offprint requests to: A. S. Brun,

e-mail: sabrun@solarz.colorado.edu
(Spiegel \& Zahn 1992); it is thought to play an important role in determining the structure and the chemical evolution of the Sun (Brun et al. 1999; Elliott \& Gough 1999).

Indeed, it appears that the solar structure deduced from helioseismology and the observed photospheric compositions can not be explained adequately without invoking some mixing in the radiative interior (Brun et al. 1999). This conclusion has been drawn after a careful study of the microscopic processes present in solar models (Christensen-Dalsgaard et al. 1996; Morel et al. 1997; Bahcall et al. 1998a; Brun et al. 1998). More precisely, in the early 90's, after significant improvements in the description of the solar plasma through better equation of state, opacities and nuclear reaction rates, helioseismic studies have established the need for microscopic diffusion of helium and heavy elements in the radiative interior (Christensen-Dalsgaard et al. 1993). But it was soon realized that models including only microscopic diffusion exhibit sharp composition gradients below the base of the convection zone which are not consistent with helioseismic data; these favour instead smoother composition profiles within this region (Basu \& Antia 1994). In spite of further improvements in solar models, this discrepancy still persists around $0.7 R_{\odot}$ 
(Christensen-Dalsgaard et al. 1996; Brun et al. 1998), suggesting that some extra mixing must be implemented in the models.

Further, using primary inversions for sound speed and density and the equations of thermal equilibrium, Antia \& Chitre (1998) have inferred the hydrogen abundance profile in the radiative interior. This profile confirms the presence of such mixing in the Sun, as the hydrogen abundance appears to be almost constant in the region $r>0.68 R_{\odot}$.

Another evidence for mixing occurring in that region comes from the photospheric light elements composition. Purely microscopic processes cannot reproduce the under abundance of lithium observed in the Sun and in open clusters (Grevesse et al. 1996; Cayrel 1998; Richard et al. 1996; Turcotte et al. 1998; Brun et al. 1999).

We are thus compelled to introduce some mixing processes in the stably stratified radiative interior. The possible causes of instabilities leading to such mixing are the solar rotation, the magnetic field or penetrative convection (Zahn 1998). The recent study of Balachandran \& Bell (1998) on the photospheric light elements abundance of ${ }^{7} \mathrm{Li}$ and ${ }^{9} \mathrm{Be}$ puts strong constraints on the extent, amplitude and location of such instabilities. It is now believed that only the lithium is significantly depleted, by more than a factor of 100 in comparison to the meteoritic composition, while the beryllium has varied by only $10 \%$ over the last 4.6 Gyr. The temperatures at which these two species are destroyed by nuclear burning are respectively $\sim 2.7 \times 10^{6} \mathrm{~K}\left(\right.$ at $0.66 R_{\odot}$ ) and $\sim 3.2 \times 10^{6} \mathrm{~K}$ (at $0.59 R_{\odot}$ ), which are relatively close to the temperature at the base of the convection zone $\sim 2.2 \times 10^{6} \mathrm{~K}$ at $0.713 R_{\odot}$ (Christensen-Dalsgaard et al. 1991). This implies that any macroscopic processes for such lithium destruction have to be located near the top of the radiation zone and cannot extend deeper than $(\sim 8 \%)$ in solar radius without producing an excessive destruction of ${ }^{9} \mathrm{Be}$.

This requirement is satisfied if the mixing is confined in the tachocline. For this reason, Brun et al. (1999) calculated the mixing occurring in that layer, based on Spiegel \& Zahn's hydrodynamical description of the tachocline. They found indeed that such mixing improved the agreement between the models and the Sun, provided the secular variation of the tachocline was taken into account. Alternative approaches based on gravity waves (Montalban \& Schatzman 1996) and magnetic field (Barnes et al. 1999) have also been studied.

In this paper we intend to go further in understanding the influence of tachocline mixing on the solar internal structure and composition by using the powerful seismic diagnostic. In particular, we examine the cumulative effect of varying, within their intrinsic uncertainties, the basic microscopic ingredients such as the nuclear reaction cross sections, the microscopic diffusion coefficients, etc. Seismic probing of the solar structure has been improved to a level where it can be used to constrain physical processes, since the Sun provides a ready-made cosmic laboratory for testing various aspects of physics. For example, there have been some attempts to constrain the nuclear reaction rate for $p p$ reaction using helioseismic data (Antia \& Chitre 1998, 1999; Degl'Innocenti et al. 1998; Schlattl et al. 1999). These studies indicate that the cross-section for $p p$ reaction needs to be increased by a few percent over the currently accepted value (Adelberger et al. 1998). Using a similar approach Weiss et al. (2001) found that enhancing the electron screening by about $5 \%$ improves the agreement between solar model and helioseismically inferred sound speed. Therefore, we would like to revise, among other quantities, the $p p$ crosssection deduced from helioseismology, by using in our solar models either the weak (Salpeter 1954) or intermediate (Mitler 1977) treatment for electron screening. We also deduce the photospheric hydrogen abundance $X_{\mathrm{ph}}$, the maximum extent $h$ of the tachocline mixing allowed in a solar model and predict the theoretical neutrino fluxes in light of the recent SNO results (Ahmad et al. 2001, 2002).

We have organised our paper as follows. In Sect. 2, we briefly recall how we compute our 1-D solar models with or without the presence of tachocline mixing, describe our inversion techniques of the solar acoustic frequencies and demonstrate the need for further progress in solar modelling. In Sect. 3, we present our latest results on the sound speed, density, hydrogen abundance and temperature profiles obtained with our modified solar tachocline models and discuss the resulting neutrino fluxes. Finally in Sect. 4, we comment on our findings and outline our conclusions.

\section{Modelling approach}

\subsection{Construction of solar models with tachocline mixing}

In order to model the Sun, we use the CESAM code (Morel 1997), which solves the structure equations (Kippenhahn \& Weigert 1994) in time and space for a spherically symmetric star of one solar mass $\left(M_{\odot}\right)$ in mechanical and thermal equilibrium. Once the evolved structure of a given model reaches the age of $4.6 \mathrm{Gyr}$, including a pre main sequence (PMS) phase of $\sim 50 \mathrm{Myr}$, it is calibrated to the solar radius $R_{\odot}$, luminosity $L_{\odot}$ and surface heavy elements abundance $(Z / X)_{\mathrm{ph}}$ to within an accuracy of $10^{-5}$ (see Table 1 ). This is done by modifying the mixing length parameter $\alpha$, the initial helium $Y_{0}$ and heavy elements $Z_{0}$ abundances. This accurate calibration of solar values is crucial and allows us to test different solutions in our search for the best agreement between our models and the Sun. We refer to Brun et al. (1998) for a more detailed description of our solar models.

The macroscopic mixing present in the tachocline is modelled by adding to the equation for chemical evolution an effective time dependent diffusion coefficient $D_{\mathrm{T}}(r, t)$, based on the hydrodynamical description of the tachocline developed by Spiegel \& Zahn (1992). In their study they invoked the anisotropy of the turbulence in a stratified medium to explain the thinness of this layer. We refer to Brun et al. (1999) for a complete description of the different steps followed to deduce from their model the effective turbulent diffusivity $D_{\mathrm{T}}(r, t)$ used in this work. This coefficient depends on two parameters:

- the tachocline thickness at the solar age, $h$ (or the closely related quantity $d \sim 2 h$ ), a relatively well-known quantity (Antia et al. 1998; Corbard et al. 1999, see Table 1),

- the Brunt-Väisälä frequency $\mathcal{N} / 2 \pi$, which varies with depth and is taken as constant in this model, representing some average over the tachocline; furthermore, it depends on the extent of overshoot and its value is therefore somewhat uncertain. 
Table 1. Solar observations: physical parameters, helioseismic observations, solar neutrino detections.

\begin{tabular}{l}
\hline \hline Physical parameters \\
$M_{\odot}=(1.9891 \pm 0.0004) \times 10^{33} \mathrm{~g}$ \\
$R_{\odot}=(6.9599 \pm 0.0002) \times 10^{10} \mathrm{~cm}$ \\
$L_{\odot}=(3.846 \pm 0.004) \times 10^{33} \mathrm{erg} \mathrm{s}^{-1}$ \\
Age $=4.6 \pm 0.04 \mathrm{Gyr}$ \\
$(Z / X)_{\mathrm{ph}}=0.0245 \times(1 \pm 0.1)$ \\
Helioseismic observations \\
$Y_{\text {surf }}=0.249 \pm 0.003$ \\
$R_{\mathrm{bcz}} / R_{\odot}=0.713 \pm 0.003$ \\
$h / R_{\odot} \leq 0.05($ tachocline thickness$)$ \\
$\mathrm{Solar}$ neutrino detections \\
${ }^{71} \mathrm{Ga}=75 \pm 5 \mathrm{SNU}($ average of all Gallium experiments $)$ \\
${ }^{37} \mathrm{Cl}=2.56 \pm 0.23 \mathrm{SNU}(\mathrm{Homestake})$ \\
$\mathrm{H}_{2} \mathrm{O}=2.32 \pm 0.08 \times 10^{6} \mathrm{~cm}^{-2} \mathrm{~s}^{-1}$ (SuperKamiokande) \\
$\mathrm{D}_{2} \mathrm{O}=1.75 \pm 0.14 \times 10^{6} \mathrm{~cm}^{-2} \mathrm{~s}^{-1}$ (SNO-charged current) \\
$\mathrm{D}_{2} \mathrm{O}=5.09 \pm 0.62 \times 10^{6} \mathrm{~cm}^{-2} \mathrm{~s}^{-1}$ (SNO-neutral current)
\end{tabular}

Note: For the gallium and chlorine detections of the solar neutrino flux we adopt the standard unit, $1 \mathrm{SNU}=10^{-36}$ captures/atom $/ \mathrm{s}$.

The time dependence of the angular velocity is based on the Skumanich law (Skumanich 1972), e.g., $\Omega(t) \propto t^{-1 / 2}$. This law is not adequate for the early phases of the solar evolution when the star contracts and/or exchanges angular momentum with its accretion disk (see Piau \& Turck-Chièze 2001). Nevertheless, for this study concerned mainly with the present day Sun, it is satisfactory.

\subsection{Inversion techniques}

To test and constrain the solar models, we compare their sound speed, density, temperature and hydrogen abundance profiles with seismically deduced ones. The sound speed and density profiles are inferred using a Regularised Least Squares (RLS) inversion technique (Antia 1996). This primary inversion is based only on the equations of mechanical equilibrium, and has been tested through extensive comparisons (e.g., Gough et al. 1996). For these primary inversions, we use a set of modes in the range of harmonic degree $\ell<190$, obtained from the first 360 days of operation of the Michelson Doppler Imager (MDI) (Schou et al. 1998). The inversion results in the central region depend on the set of low degree acoustic modes used (i.e., $\ell \leq 2$, Basu et al. 2000) but not to the extent of modifying the conclusions of this work. To infer secondary quantities such as temperature and chemical composition within the Sun, we follow the treatment given by Antia \& Chitre (1998).

Apart from evolutionary solar models, we also construct some static ones, using a composition profile calculated by the evolutionary stellar structure code CESAM. These models use the same physical inputs as the evolutionary models, but include a different treatment of atmosphere, using the atmospheric model of Vernazza et al. (1981) as well as the opacity tables from Kurucz (1991) at low temperatures and the formulation of Canuto \& Mazzitelli (1991) to calculate the convective flux. Because of these differences the surface layers in the Sun are better represented in these static models.

\subsection{Earlier results}

Before introducing any new modifications in our evolutionary solar models, let us recall what are the strengths and weaknesses of the tachocline models of Brun et al. (1999).

In Fig. 1 we represent the relative sound speed and density differences $\delta c / c$ and $\delta \rho / \rho$ between the seismic Sun obtained using the inversion procedure described above and our purely microscopic diffusive model, hereafter referred to as ref model or a typical tachocline model of Brun et al. (1999), namely, model $B_{t z}$. A first quick look reveals that the mixed model $B_{t z}$ shows a better overall agreement both for density and sound speed compared to the reference one. For both these quantities, the transition at the base of the homogeneous convection zone is smoother, resulting in an almost disappearance of the pronounced peak seen in $\delta c / c$ of model ref. Further, the hydrogen abundance in the convective envelope is slightly closer to the seismic one, due mainly to the limiting action of the macroscopic mixing on the gravitational settling of the chemical elements (see Table 3). Brun et al. (1999) found that macroscopic mixing at the base of the convection zone reduces by $25 \%$ the microscopic diffusion in comparison to a purely microscopic model such as ref. Moreover, as we shall see in Sect. 3.3, the hydrogen abundance profile is smoother and does not exhibit any sharp gradient just below the base of the convection zone. Further, the lithium depletion achieved in the mixed model is significant, of the order of 100 , and follows quantitatively well

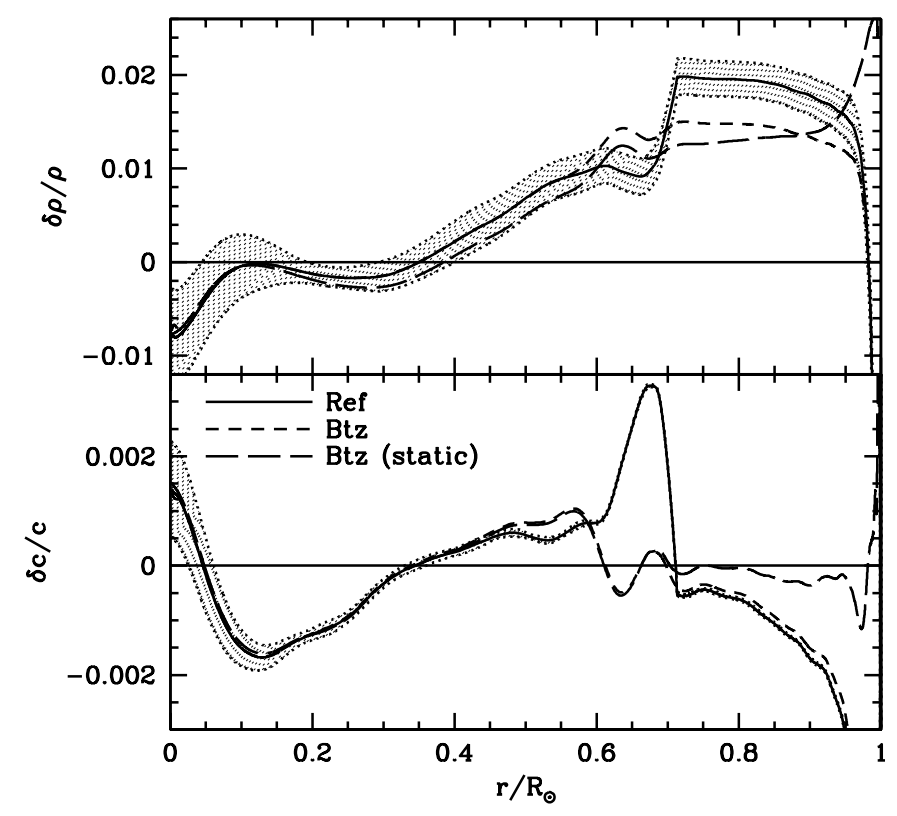

Fig. 1. The relative difference in sound speed and density profiles between the Sun and solar models is shown as a function of fractional radius. Model ref including only microscopic diffusion is represented with a solid line and the mixed models $B_{t z}$ and its static equivalent with respectively a short-dashed and long-dashed lines. Superimposed on model ref is the $1 \sigma$ error envelope coming from the helioseismic inversion. 
the open cluster observations, such as in the Hyades, or older clusters such as NGC 752. On the other hand, model ref burns its lithium mainly in the PMS phase with only a tiny fraction being depleted during the main sequence evolution due to gravitational settling.

In Fig. 1 we also compare the sound speed and density profiles of a typical static model which has been constructed using the same physical inputs as in model $B_{t z}$ including its composition profiles as well. In the interior, the sound speed of the static model is almost the same as that of the evolutionary model, while in the outer layers it represents better that of the Sun. This improvement is most probably due to better treatment of surface layers in the static model through the use of a different prescription to calculate convective flux and also the adoption of a better atmospheric model.

Despite all the positive aspects, solar models including tachocline mixing still need further improvements. Model $B_{t z}$, for example, shows significant departure from the seismic Sun in its density profile, even though this quantity is in better agreement in comparison to model ref. Further, it assumes a photospheric value for the heavy elements, $(Z / X)_{\mathrm{s}} \sim 0.0255$ which is somewhat higher than the observed value. The reason is that a model calibrated to $(Z / X)_{\mathrm{s}}=0.0245$, such as model $B_{t}$ in Brun et al. (1999), is not as close to the seismic Sun as model $B_{t z}$, because of a smaller $Z$ content and to the resulting variation of the opacities in the radiative interior. Therefore, we would like to reach between our new models and the Sun an agreement in density and sound speed better than that with model $B_{t z}$, but without having to relax the $(Z / X)_{\mathrm{s}}$ constraint in the calibration process. Finally, the lithium depletion in the PMS phase is overestimated, indicating the need for a better treatment of this early phase of evolution. All the cited improvements can come both from a better treatment of the mixing at the top of the radiation zone or by a better microscopic description. We refer to Piau \& Turck-Chièze (2001) for a careful study of the PMS lithium depletion problem and focus our attention on the present Sun. We propose to keep for most of our models the same treatment for the tachocline mixing as introduced in Brun et al. (1999) but to allow for variations within uncertainties of the main physical ingredients, in order to see if any improvements can be obtained before introducing a new description of the tachocline mixing.

\section{Improved solar models}

\subsection{Model parameters}

We see from the foregoing discussion that there is still need to improve our mixed tachocline models. In this section we outline the modifications and models computed for this study, which for the sake of clarity are also summarised in Table 2 with their designation and the corresponding choice of parameters.

- $\quad p p$ nuclear reaction: when dealing with the properties of the solar core one is inevitably led to the nuclear reaction rates and their intrinsic uncertainties (Brun et al. 1998; Bahcall et al. 1998a; Morel et al. 1999). Unfortunately, the fundamental nuclear reaction in the $p p$-chain, i.e., $p+p \rightarrow \mathrm{D}+\mathrm{e}^{+}+v$, is an

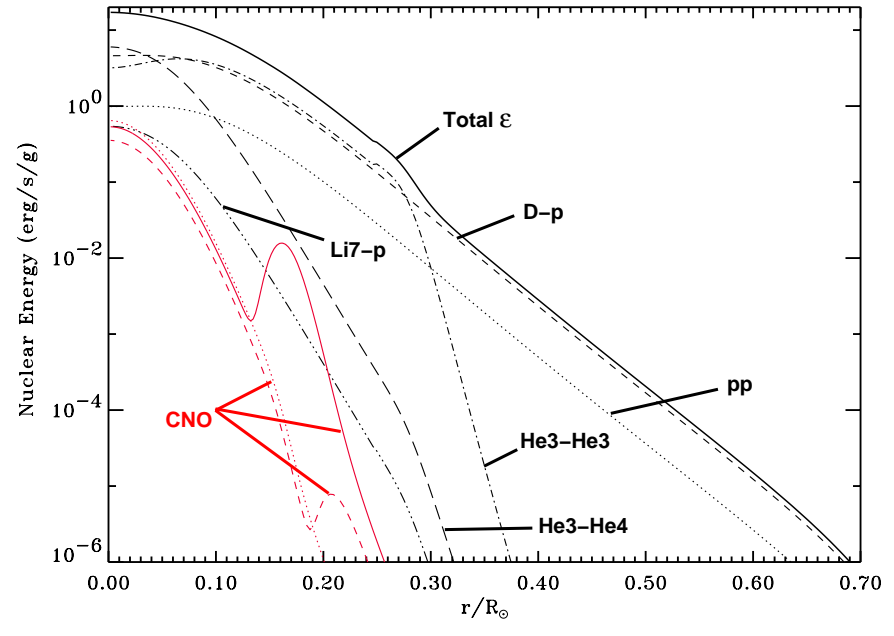

Fig. 2. Contribution to the nuclear energy generation of the main $p p$ chain and $\mathrm{CNO}$ cycles nuclear reactions as a function of the normalised solar radius.

electroweak interaction and its cross section has not been determined via direct experimentation. Recent theoretical works give an uncertainty of about 1-2\% in the determination of $S_{11}$ (Adelberger et al. 1998). But a seismic calibration of this cross section indicated that up to a $4 \%$ increase is favoured (Antia \& Chitre 1998). We have therefore decided to allow in our models for a variation up to $4 \%$ of this dominant cross-section and will also determine which value of $S_{11}(0)$ gives the best agreement with helioseismic inversion.

Aside the fundamental $p p$ nuclear reaction, other reactions can possibly modify the central structure of the Sun. In Fig. 2 we plot the energy production of the dominant nuclear reactions from the $p p$ chains as well as the three main reactions from the $\mathrm{CNO}$ cycle. These reactions are the following: $\mathrm{D}(p, \gamma){ }^{3} \mathrm{He},{ }^{3} \mathrm{He}\left({ }^{3} \mathrm{He}, 2 \mathrm{p}\right){ }^{4} \mathrm{He},{ }^{3} \mathrm{He}(\alpha, \gamma){ }^{7} \mathrm{Be},{ }^{7} \mathrm{Li}(p, \alpha){ }^{4} \mathrm{He}$ from the $p p$ chains and ${ }^{13} \mathrm{C}(p, \gamma){ }^{14} \mathrm{~N},{ }^{14} \mathrm{~N}(p, \gamma){ }^{15} \mathrm{O}\left(\mathrm{e}^{+} v\right){ }^{15} \mathrm{~N}$, ${ }^{15} \mathrm{~N}(p, \alpha){ }^{12} \mathrm{C}$ from the $\mathrm{CNO}$ cycle. To plot these curves we have used the internal structure of ref model for the present Sun.

Not all the nuclear reactions displayed in Fig. 2 are expected to modify the thermal structure of the solar core. We can already discard some of them by considering either their importance in the energy budget or the time they require to reach equilibrium.

- CNO cycle nuclear reactions: because the Sun is a low mass star, we don't expect the CNO nuclear reactions to significantly influence the solar central region since the cycle contributes to less than $2 \%$ of the total nuclear energy production (Clayton 1968; Bahcall 1989; Bahcall et al. 2001). We have therefore not introduced any modification of these reactions in our models and refer to Turck-Chièze et al. (2001) for a discussion of their influence on the solar structure.

- $\quad$ D- $p$ and ${ }^{7} \mathrm{Li}-p$ nuclear reactions: because the lifetime of deuterium and lithium is very short, these elements quickly reach equilibrium abundance in the temperature and density range prevailing in the solar core. Consequently, these reactions do not have a significant impact on the core structure even though they are very energetic. We refer to Gautier \& Morel (1997) for a discussion of the important $\mathrm{D} / \mathrm{H}$ astrophysical 
Table 2. Model parameters.

\begin{tabular}{|c|cc|cccccc|}
\hline \hline & \multicolumn{2}{|c|}{ Mixing } & \multicolumn{7}{|c|}{ Microscopic Variations } \\
Models & $d / R_{\odot}$ & $\mathcal{N} / 2 \pi$ & $(Z / X)_{s}$ & $S_{11}$ & $S_{33}$ & $S_{34}$ & $f_{\text {sc }}$ & $D_{i}$ \\
\hline \hline$R e f$ & - & - & 0.0245 & - & - & - & I & - \\
$B_{t z}$ & 0.1 & 25 & 0.0255 & - & - & - & I & - \\
\hline \hline$N 0$ & 0.1 & 25 & 0.0245 & $+2.0 \%$ & - & - & I & - \\
$N 02$ & 0.1 & 25 & 0.0245 & $+2.0 \%$ & - & $-10 \%$ & I & - \\
$N 03$ & 0.1 & 25 & 0.0245 & $+2.0 \%$ & $-8 \%$ & $+10 \%$ & I & - \\
$N 0 W$ & 0.1 & 25 & 0.0245 & $+2.0 \%$ & - & $-10 \%$ & W & - \\
$N$ & 0.1 & 25 & 0.0245 & $+3.5 \%$ & - & - & I & - \\
$N 1$ & 0.1 & 25 & 0.0245 & $+3.5 \%$ & $+8 \%$ & - & I & - \\
$N 2$ & 0.1 & 25 & 0.0245 & $+3.5 \%$ & - & $-10 \%$ & I & - \\
$N D$ & 0.1 & 25 & 0.0245 & $+3.5 \%$ & - & $-10 \%$ & I & $-10 \%$ \\
$N M$ & 0.15 & 25 & 0.0245 & $+3.5 \%$ & - & $-10 \%$ & I & - \\
$N E$ & 0.04 & - & 0.0245 & $+3.5 \%$ & - & $-10 \%$ & I & - \\
\hline
\end{tabular}

Note: The parameters, $d$ and $\mathcal{N} / 2 \pi$, represent twice the extent of the tachocline $h$ and the Brunt-Väisälä frequency (in $\mu \mathrm{Hz}$ ) in the overshoot region and are related to our effective macroscopic coefficient $D_{\mathrm{T}}$ (cf., Brun et al. 1999). $(Z / X)_{\mathrm{s}}, S_{11}, S_{33}, S_{34}, f_{\mathrm{sc}}$ and $D_{i}$ are respectively, the surface ratio of heavy elements to hydrogen abundances of the models at the solar age, the variation applied in the nuclear cross section of $p p,{ }^{3} \mathrm{He}-{ }^{3} \mathrm{He}$ and ${ }^{3} \mathrm{He}-{ }^{4} \mathrm{He}$, the screening prescription used in the model (either Weak or Intermediate) and the variation of the microscopic diffusive coefficient.

ratio and to Brun et al. (1999) for a study of the influence of the ${ }^{7} \mathrm{Li}-p$ cross section on photospheric lithium abundance. We will adopt in all our models the cross sections proposed by the NACRE compilation (Angulo et al. 1999).

- $\quad{ }^{3} \mathrm{He}-{ }^{3} \mathrm{He}$ and ${ }^{3} \mathrm{He}-{ }^{4} \mathrm{He}$ nuclear reactions: these nuclear cross sections play a crucial role in determining the branching between the $p p \mathrm{I}$ and the $p p \mathrm{II}$ and $p p \mathrm{III}$ chains and thus directly influence the high energy neutrino production. ${ }^{3} \mathrm{He}-{ }^{3} \mathrm{He}$ is one of the most energetic reactions in the $p p$-chain along with $\mathrm{D}-p$. Unlike deuterium and lithium, ${ }^{3} \mathrm{He}$ does not reach its equilibrium value on a very short time scale but instead slowly builds up for temperatures less than $8 \times 10^{6} \mathrm{~K}$ (Clayton 1968). In a solar model, the resulting theoretical ${ }^{3} \mathrm{He}$ abundance profile peaks around $r=0.28 R_{\odot}$ with a characteristic bell-like curve due to the competition respectively between its creation and its destruction in the outer and in the inner regions of the solar core. At the same time, being one of the most energetic reactions in the $p p$ chains and reaching equilibrium gradually in the outer parts of the nuclear region, this cross section is expected to have an influence on the thermal structure of the solar core at a level where seismic inversions can detect it. Even though the ${ }^{3} \mathrm{He}-{ }^{4} \mathrm{He}$ nuclear reaction does not contribute much to the solar energy budget, the fact that it involves both ${ }^{3} \mathrm{He}$ and ${ }^{4} \mathrm{He}$ chemical elements makes it also an important reaction to study. The experimental uncertainty of $S_{33}$ and $S_{34}$ are respectively $\pm 8 \%$ and $\pm 10 \%$ (Adelberger et al. 1998).

- Intermediate screening: we use the intermediate screening prescription of Mitler (1977) in all our models except one that uses the classical weak screening of Salpeter (1954). It should be recognised that the screening in stellar nuclear reaction rates is a sensitive issue which is not yet completely understood (Dzitko et al. 1995; Wilets et al. 2000). Depending on the solar thermodynamical conditions and the chemical species interacting in the nuclear reaction considered, one has to introduce the adequate screening factor, $f_{\mathrm{sc}}$, coming from the surrounding particles present in the solar plasma which in general differ from the screening effect evaluated by nuclear physicist in their experiments. There are thus several sources of uncertainties in evaluating the cross sections and screening effects for any given nuclear reaction that we intend to consider in this work.

- Opacities, microscopic diffusion and heavy elements abundance $Z$ : the structure of solar radiative zone is very sensitive to these two physical processes and to the heavy elements abundance. These are all closely related since a change in $Z$ leads to a change in the opacity $\kappa$ and in the microscopic diffusion, which in turn modify $Z$ as a consequence of the iterative calibration process. The opacity is accurately computed (error $\sim 5 \%$ ) for temperatures greater than $10^{4} \mathrm{~K}$ (Iglesias \& Rogers 1996), their main sources of uncertainties come from the relative composition and the ionisation degree of the heavy elements as well as quantum effects (Rogers \& Iglesias 1998). In this work we will leave $\kappa$ unchanged and will concentrate instead on the heavy elements abundance $Z$ and on the amplitude of the microscopic coefficients $D_{i}$. From a detailed comparison with the work of Turcotte et al. (1998), Brun et al. (1998) have confirmed that the analytical expressions for the microscopic diffusion coefficients given by Michaud \& Proffitt (1993) are accurate enough to deal with the solar case. For the Sun, these uncertainties are at most $15 \%$. In order to reduce the chemical composition gradient present at the base of the convection zone, which has been found to be too large in model ref compared to the seismically deduced one, we have run one model with $D_{i}$ reduced by $10 \%$. The heavy elements abundance of our models has been calibrated to the observed value of Grevesse et al. (1996), i.e., $(Z / X)_{\mathrm{ph}}=0.0245$ (see Table 1). Model $B_{t z}$ introduced earlier has been computed with an initial heavy elements abundance $Z_{0}=0.01959=Z_{\text {ref }}$ that leads to $(Z / X)_{\mathrm{s}}=0.0255$ or $4 \%$ higher than $(Z / X)_{\mathrm{ph}}$.

- Mixing: it is very tempting to introduce a mild mixing in the nuclear region, for example very close to the ${ }^{3} \mathrm{He}$ peak, in order to improve the solar model structure and to reduce 
the predicted neutrino fluxes (Haxton 1997; Brun et al. 1998). However, the presence of a mixing in the solar core can be rejected on account of the helioseismic constraints, due to the huge disagreement in the central region that it generates (of the order of few $\%$ in $\delta c / c$ ). This seismic evidence along with the recent results of the SNO neutrino experiment (Ahmad et al. 2001, 2002), strongly disfavour a "macroscopic mixing" as the source of the electron neutrino deficit seen on Earth's detectors but instead support the idea of neutrino flavour oscillations.

Therefore we limited ourselves to the tachocline region for which we have better evidence for mixing and a relatively more elaborate physical description available. We used the parameters of model $B_{t z}$ (e.g., $d=0.10 R_{\odot}$ and $\mathcal{N} / 2 \pi=25 \mu \mathrm{Hz}$ ), which have been proven to give a reasonable agreement with seismic constraints and light elements photospheric abundance (cf. Sect. 2.3 and Brun et al. 1999). For one case we have assumed a wider mixing zone, i.e., $d=0.15 R_{\odot}$, in order to limit even more the gravitational settling and therefore reduce the steep gradients seen in the sound speed, density and composition profile at the base of the convection zone. We have also used the prescription introduced by Elliott \& Gough (1999), namely a constant diffusion coefficient $D_{\mathrm{TE}}$ operating over a small domain $d=0.04 R_{\odot}$. It is not our intention to reevaluate this coefficient, but just to compare both mixing prescriptions.

Having introduced all these modifications in our evolution code we derive the profiles of sound speed, density, hydrogen abundance and temperature that we discuss in the following subsections.

\subsection{Inferred sound speed and density profiles}

We first consider the relative differences in the sound speed and density between the Sun and our new modified solar models.

\subsubsection{Increasing the pp cross section}

In Fig. 3 we display two new models, $N 0$ and $N$, which include respectively an enhanced $p p$ nuclear cross section by $2 \%$ and $3.5 \%$, along with the older models $r e f$ and $B_{t z}$ already presented in Fig. 1. Clearly the relative differences in the sound speed and density between the Sun and model $N$, and to a lesser extent model $N 0$, are smaller in comparison to model ref and the mixed model $B_{t z}$. This is really encouraging because contrary to model $B_{t z}$, both models $N 0$ and $N$ have been calibrated to the exact value of $(Z / X)_{\mathrm{s}}=0.0245$ used in model $r e f$. This indicates that the effect of $Z$ via the opacity $\kappa$ can be compensated by a small variation of the $S_{11}$. Thus a variation of the order of a few percent of either the heavy elements abundance or the fundamental $p p$ nuclear reaction rate seems to have the same effect on the model sound speed profile in the upper part of the radiative region but not below $r=0.3 R_{\odot}$ where contrary to case $B_{t z}$, model $N$ departs from ref. For the density, a variation of $S_{11}$ modifies the profile everywhere resulting in a significantly better agreement for case $N$, at least above $r=0.2 R_{\odot}$. Thus it is very useful to assess the accuracy of a model by considering both the sound speed and the

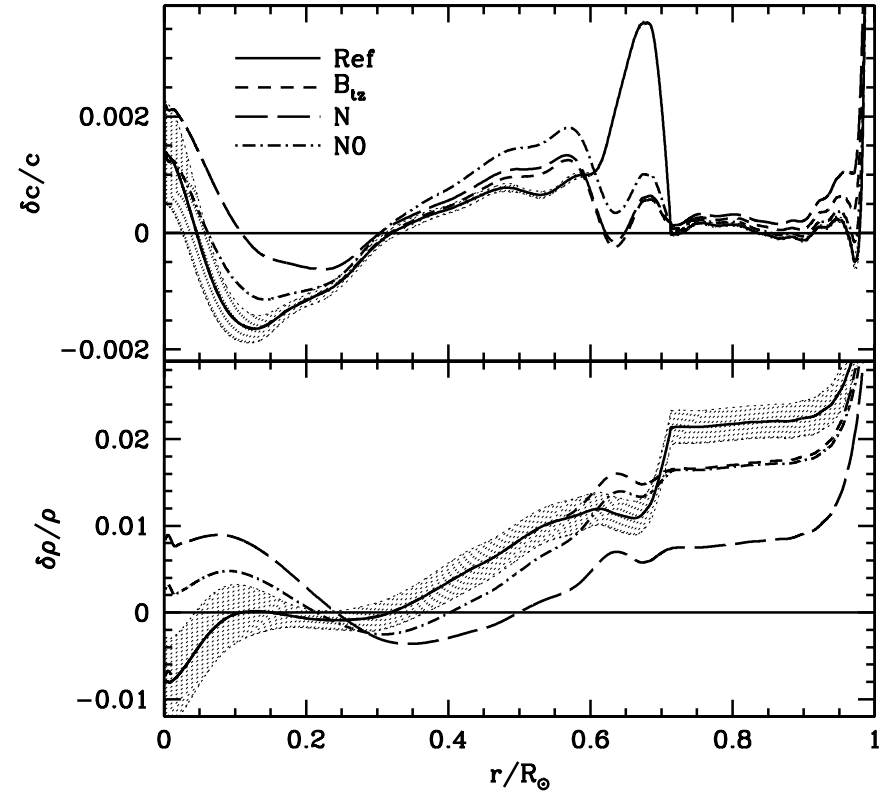

Fig. 3. The relative difference in sound speed and density profiles between the Sun and solar models as a function of fractional radius. Model ref including only microscopic diffusion and the mixed models $B_{t z}, N 0$ and $N$ (the latter two with increased $p p$ cross section), are represented respectively with solid, short-dashed, dash-dotted and long-dashed lines. Superimposed on model ref is the $1 \sigma$ error envelope coming from the helioseismic inversion.

density profiles, because it allows one to distinguish the impact of different physical processes on solar structure. In this particular case an increase of $S_{11}$ by $3.5 \%$ seems to be favoured by helioseismology, as opposed to an increase of $S_{11}$ by $2 \%$ or of $(Z / X)_{s}$.

It may be noticed that in Fig. 3 the agreement in sound speed inside the convection zone is much better as compared to that for the same models in Fig. 1. This improvement arises because we have scaled the solar radius in the models by a factor of 1.0003 before forming the difference with the seismically deduced sound speed and density profiles. This scaling of the radius appears to remove most of the discrepancy in the upper convection zone, but does not affect the interior. This may be expected since a correction of $0.03 \%$ in radial distance is only a small fraction of scale height in the interior, while it can become comparable to the scale height in the photospheric layers, resulting in significant differences in outer regions. A better agreement with the Sun is also obtained in the outer convective zone in the case of a static model (Fig. 1), which assumes a different treatment of the surface layers. It would appear that uncertainties in treatment of these layers are responsible for the discrepancy in the outer convection zone. In standard solar models the surface is normally defined as the layer where the temperature equals the effective temperature. Because of significant uncertainties in treatment of surface layers, the position of the surface may not be correctly estimated in a solar model. Thus, we believe that the scaling of radius effectively corrects for this error. In all subsequent figures we have used this scaled radius when comparing the solar models with profiles inferred from inversions. 


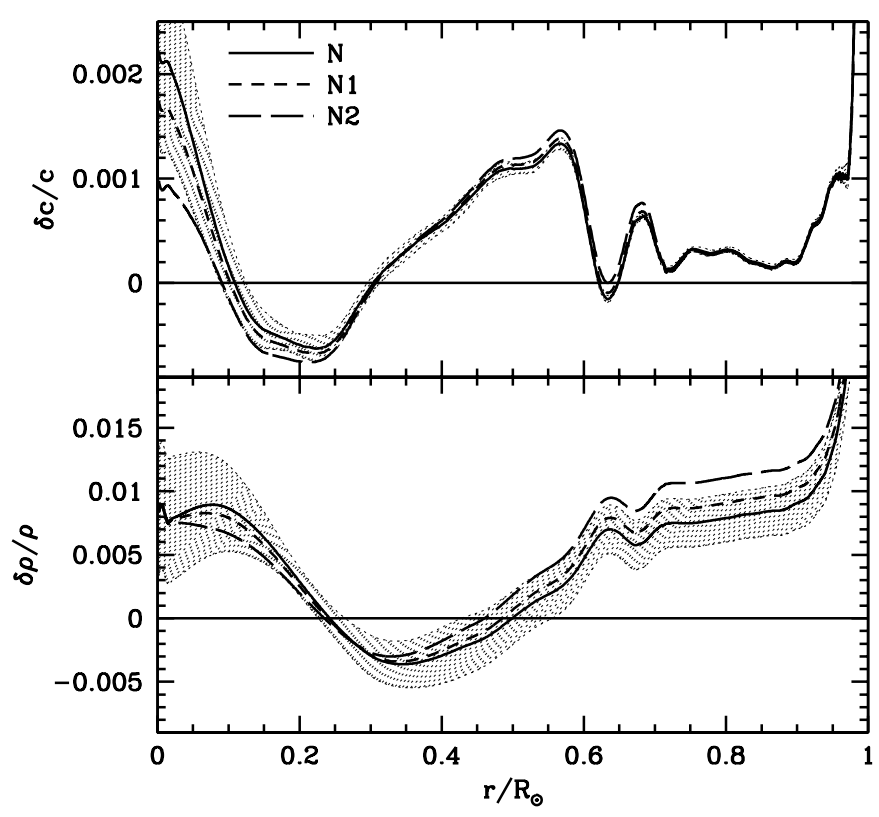

Fig. 4. The relative difference in sound speed and density profiles between the Sun and solar models as a function of fractional radius, showing the effect of modifying the cross sections $S_{33}$ and $S_{34}$. The mixed models $N, N 1$ and $N 2$ are represented respectively with solid, short-dashed and long-dashed lines. Superimposed on model $N$ is the $1 \sigma$ error envelope coming from the helioseismic inversion.

\subsubsection{Modifying the ${ }^{3} \mathrm{He}-{ }^{3} \mathrm{He}$ and ${ }^{3} \mathrm{He}-{ }^{4} \mathrm{He}$ cross sections}

We have just seen that a small change in $S_{11}$ can significantly improve the agreement between solar models and the seismic Sun when combined with a treatment of the solar tachocline. In the same spirit we have computed a sequence of models including modifications of $S_{33}$ and $S_{34}$ nuclear reaction cross sections (cf. Sect. 3.1). Models $N 1$ and N2 share the same tachocline macroscopic treatment, calibration of heavy elements to $(Z / X)_{\mathrm{s}}=0.0245$ and increase of $S_{11}$ by $3.5 \%$ as model $N$, but differ by having respectively an increase of $S_{33}$ by $8 \%$ and a decrease of $S_{34}$ by $10 \%$ (see Table 2).

In Fig. 4 we plot the relative differences in density and sound speed between the Sun and models $N, N 1$ and $N 2$. We first notice that the applied modifications of the cross section $S_{33}$ and $S_{34}$ improve the core structure both in density and sound speed. A variation of $S_{34}$ by $-10 \%$ seems to affect more the very central region than a variation of $S_{33}$ by $+8 \%$ does, even if we take into account the fact that $S_{33}$ has been varied by a smaller amount. For model $N 2$ the agreement in $\delta c / c$ in the core improves by a factor 2 with respect to model $N$, whereas for model $N 1$ it does only by $20 \%$ or so. For $\delta \rho / \rho$, the influence of these two cross sections is more modest and results in a small gradual change of the profile over the solar radius.

We now turn to models $N 02$ and N03 displayed in Fig. 5 along with model $N 0$ as reference. Models N02 and NO3 are identical to model $N 0$ except that they respectively include a decrease of $S_{34}$ by $10 \%$ and the cumulative opposite variations of the nuclear cross sections $S_{33}$ by $-8 \%$ and $S_{34}$ by $+10 \%$ (see Table 2), in order to modify the $p p$ branching ratio such as

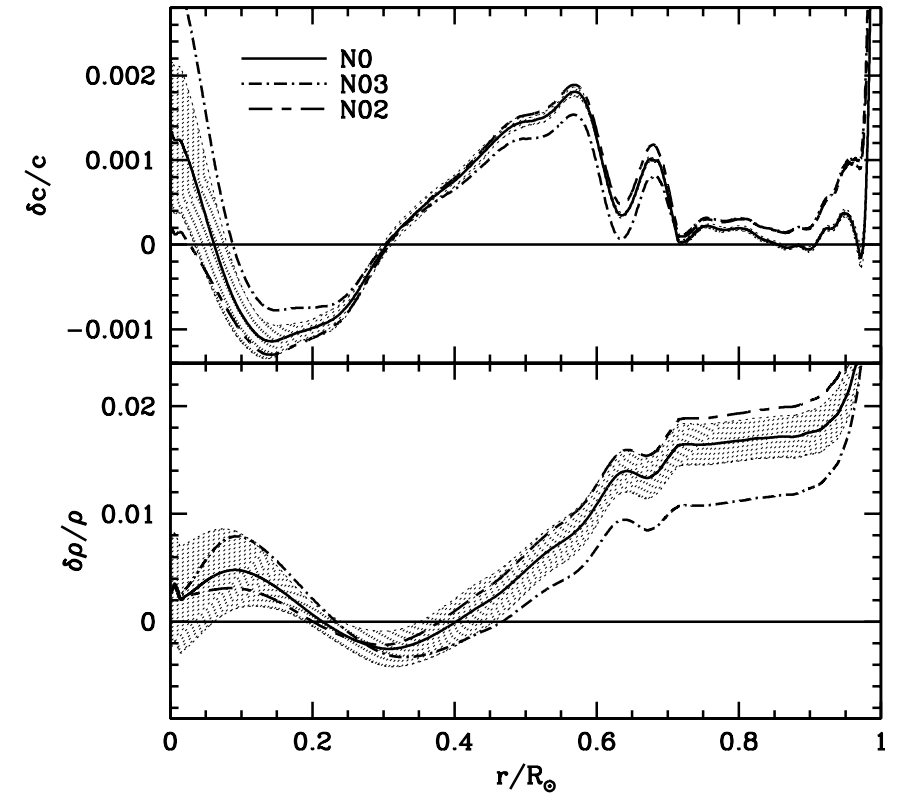

Fig. 5. The relative difference in sound speed and density profiles between the Sun and solar models as a function of fractional radius, showing the effect of modifying the cross sections $S_{33}$ and $S_{34}$. The mixed models $\mathrm{NO}, \mathrm{NO2}$ and $\mathrm{NO3}$ are represented respectively with solid, short-dash-long-dashed and dash-dotted lines. Superimposed on model $N 0$ is the $1 \sigma$ error envelope coming from the helioseismic inversion.

to increase the high energy neutrino flux. Model N02 exhibits a better core profile than model $N 0$ does by having both relative differences closer to zero. However, these two models do not differ from each other as much as their counterpart models $N$ and $N 2$ do and are within the $1 \sigma$ error bar. In overall the decrease of $S_{34}$ represents in this case a small progress toward a better agreement with the seismic data. On the contrary, model N03 that has been computed on purpose with opposite variations of $S_{33}$ and $S_{34}$ is almost everywhere in better agreement than model $N O$ is. Its density profile is significantly closer to the Sun. The main exception is in the very central part of the solar core, where the $\delta c / c$ is quite off and the $\delta \rho / \rho$ exhibits a pronouncedly curved shape. Model N03 is an interesting solar model but our variation of the $S_{33}$ and $S_{34}$ cross sections is certainly too large and goes in the wrong direction for the central parts.

In summary, these new sets of results confirm that it is possible to improve the overall agreement between the models and the seismic Sun by modifying, within their uncertainties, the rates of important nuclear reactions such as $S_{33}$ and $S_{34}$. Here it appears that the seismic data favour a decrease of $S_{34}$ and an increase of $S_{33}$ rather than the opposite.

\subsubsection{Nuclear screening}

We would like now to characterise the influence of the nuclear screening on the solar model sound speed and density profiles. We have therefore changed the screening prescription from intermediate to weak (cf. Sect. 3.1), on the top of all the modifications already introduced in one model, that we have 


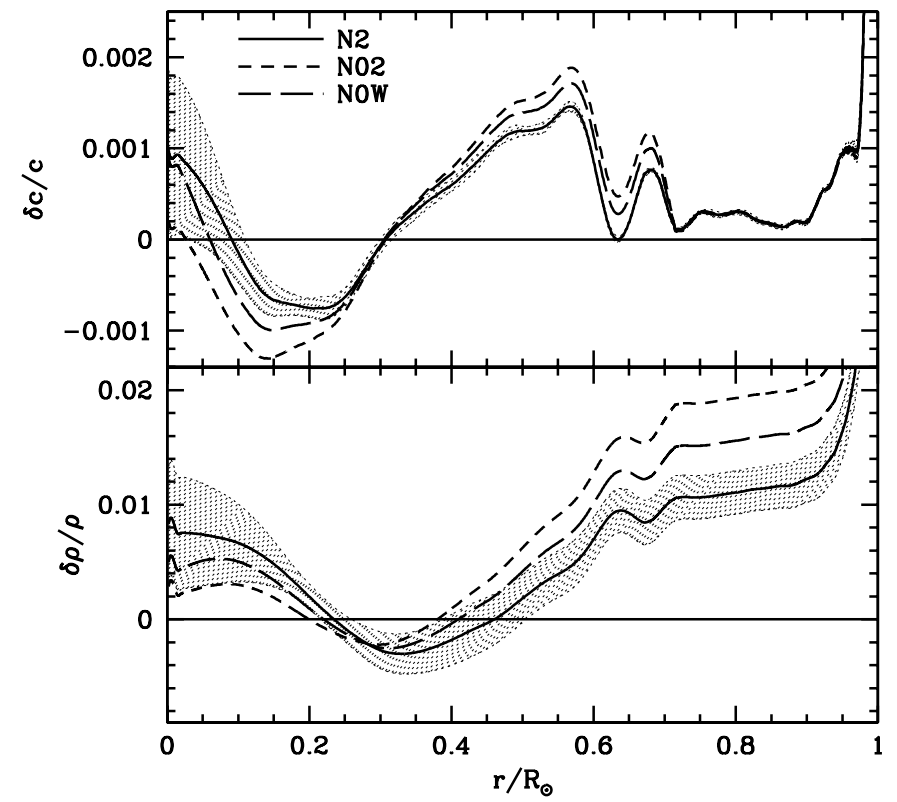

Fig. 6. The relative difference in sound speed and density profiles between the Sun and solar models as a function of fractional radius, showing the effect of changing the nuclear screening. The mixed models $N 2, N 02$ and NOW are represented respectively with solid, shortdashed and long-dashed lines. Superimposed on model $N 2$ is the $1 \sigma$ error envelope coming from the helioseismic inversion.

chosen to be $N 02$, and called that new model $N 0 W$. In Fig. 6 we compare results obtained for models $N 2, N 02$ and $N 0 W$, in order to quantify the influence of the nuclear screening on the solar core. In the very central part of model $N 0 \mathrm{~W}$ the agreement seems to not be as good as in model $N 02$. The relative sound speed difference profile between the Sun and model $N 0 W$ for $r<0.2 R_{\odot}$ reaches a value of the same order as model $\mathrm{NO}$ in Fig. 5 and thus the change of nuclear screening seems to compensate the change by $10 \%$ in $S_{34}$ made in model N02. The density profile of model $N O W$ is in better agreement with the Sun than model N02 above $0.3 R_{\odot}$. We should be careful in evaluating the effect on the core structure of using a different screening prescription, because model $N 0 \mathrm{~W}$ includes an increase of $S_{11}(0)$ already calibrated on the intermediate screening, but the inversion seems to indicate that a smaller increase of $S_{11}$ is favoured if one has to use a weak nuclear screening instead (cf., Table 3 ).

\subsubsection{Microscopic diffusion}

As already stated in Sect. 3.1, we are interested in a reduction of the steep composition gradient at the base of the convection zone, which implies a decrease of the microscopic diffusion coefficients $D_{i}$. Model $N D$ includes such a decrease by $10 \%$ of $D_{i}$ along with other variations identical to model $N 2$. It is obvious from Fig. 7, that a reduction of microscopic diffusion is not appropriate, since model $N D$ systematically departs more from the seismic Sun in the radiation zone than model N2. It is well known that microscopic diffusion modifies and improves the stratification in the radiation zone

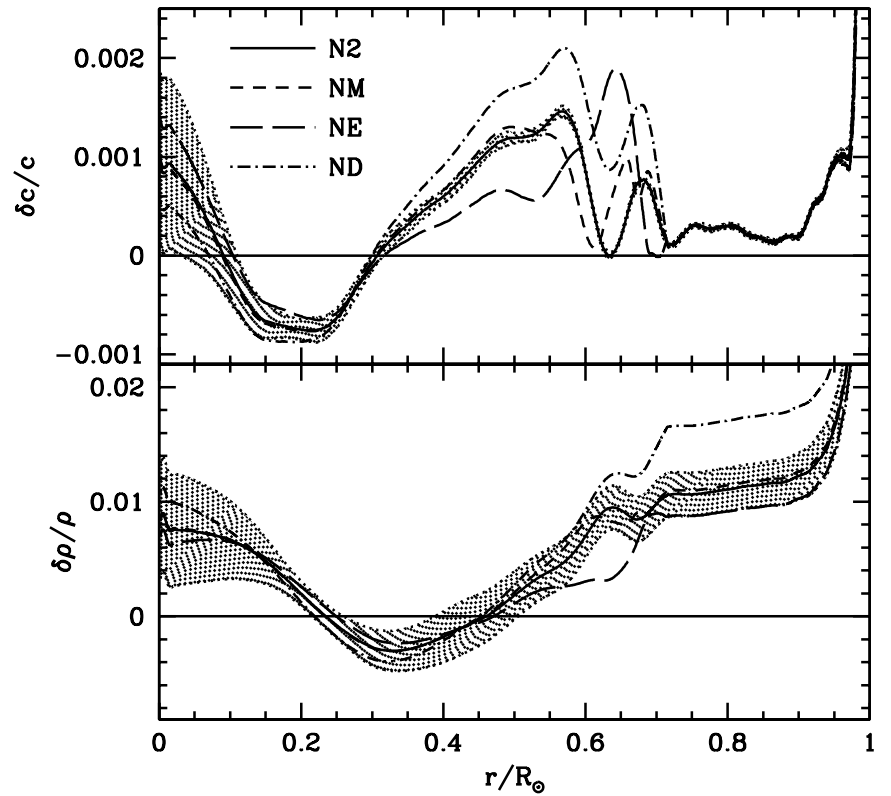

Fig. 7. The relative difference in sound speed and density profiles between the Sun and solar models. The mixed models $N 2, N M, N E$ and $N D$ are represented respectively with solid, short-dashed, longdashed and dash-dotted lines. Superimposed on model $N 2$ is the $1 \sigma$ error envelope coming from the helioseismic inversion.

(Christensen-Dalsgaard et al. 1993). Based on the sound speed and density profiles, an increase of $D_{i}$ is favoured rather than a decrease as in model $N D$. However an increase of $D_{i}$ would make the composition gradient at the base of the convective zone even steeper. Thus it seems quite unlikely that the microscopic diffusion is the remaining source of the discrepancies seen in a solar model like $N 2$.

\subsubsection{Adjusting the tachocline mixing}

In the sequence of models we have just discussed, we were only concerned with the influences on the solar structure of variations in the nuclear and atomic input data. We are now interested in assessing the impact on $\delta c / c$ and $\delta \rho / \rho$ of varying the macroscopic parameters used for the solar tachocline. In Fig. 7 we also display models $N M$ and $N E$ which include the same microscopic ingredients as model $N 2$ but with respectively, a broader mixing region with $d \sim 0.15 R_{\odot}$ (i.e., tachocline thickness $h \sim 0.075 R_{\odot}$ ) and another macroscopic treatment for the tachocline (Elliott \& Gough 1999), over a shorter distance $h \sim 0.02 R_{\odot}$ (see Table 2). We clearly see that model $N E$ does not reduce the bump in $\delta c / c$ or the steep gradient in $\delta \rho / \rho$ at the base of the convection zone seen in model ref in Fig. 3 as much as the two other models $N 2$ and $N M$. Even though model $N E$ includes the same nuclear cross section modifications, it is further away from the seismic Sun. One reason for this poorer agreement is that the tachocline mixing is too shallow. The value of $0.02 R_{\odot}$ adopted in model $N E$ for the extent of the mixing has been calibrated by Elliott \& Gough using a static model of the Sun at the present age and by convolving with the inversion kernels afterward. This convolution procedure using the inversion kernels makes the effective thickness 
of the mixing broader, mainly because of their overlapping radial resolution, and as a consequence Elliott \& Gough found that $\sim 0.02 R_{\odot}$ was large enough to get rid of the bump in $\delta c / c$. It is quite puzzling that the introduction of their mixing in our evolutionary model does not give at all the same result. Indeed model $N E$ is significantly different from models $N 2$ or $N M$, that we believe are in quite good agreement with the seismic Sun. It can be seen in Fig. 7 that an increase of the extent of the mixing region in model $N M$ does not affect that much the density and sound speed profiles except for the slight modification close to the base of the convection zone. We therefore conclude that not all prescriptions for "tachocline mixing" give the same result and that ours is quite efficient in suitably modelling this transition region.

\subsection{Hydrogen abundance profile and photospheric composition}

We have so far addressed the question of the influence of mixing and microscopic variations on the primary inversion quantities such as the sound speed and the density. We would now like to assess what are the consequences of such changes on the profile and photospheric value of the hydrogen abundance $X_{\mathrm{ph}}$. Using the secondary inversion procedure introduced by Antia \& Chitre (1998), we have compared the hydrogen abundance profile in the Sun with that predicted by our new set of solar models. By assuming the $Z$ profile of the models we have calculated the difference of $X$ between each model and the Sun.

In Fig. 8 we represent the absolute difference in hydrogen abundance profile $\delta X$ between the solar models $r e f, B_{t z}, N 0$ and $N$ (cf., Table 2) and the Sun. As with $\delta c / c$ and $\delta \rho / \rho$ displayed in Fig. 3, it is quite clear that the mixed models are closer to the inferred solar hydrogen abundance than the purely microscopic model ref is. This improvement occurs mainly close to the base of the convection zone and in the convection zone itself (i.e., indicating a closer photospheric value) as expected by the introduction of our shallow tachocline mixing. The reason for such an improvement is twofold:

- Firstly, by introducing a macroscopic mixing at the base of the convection zone, we hinder the gravitational settling of the chemical species and as a result there are relatively more helium and heavy elements in the convection zone, thus reducing the hydrogen contribution in the plasma composition mixture to a value closer to the seismically inferred one.

- Secondly, the existence of an extended/mixed plateau of the chemical composition due to the presence of a macroscopic mixing at the base of the convection zone is in better agreement with the seismically inferred $X$ profile.

This result, along with the improved sound speed and density profiles discussed in the previous subsection, confirms the presence of macroscopic mixing at the base of the convection zone and the necessity to introduce this process in solar models. Figure 8 also reveals that a small increase by less than $4 \%$ of the cross-section of the fundamental nuclear reaction $p p$ is favoured as well. Indeed models $N 0$ and $N$ are significantly

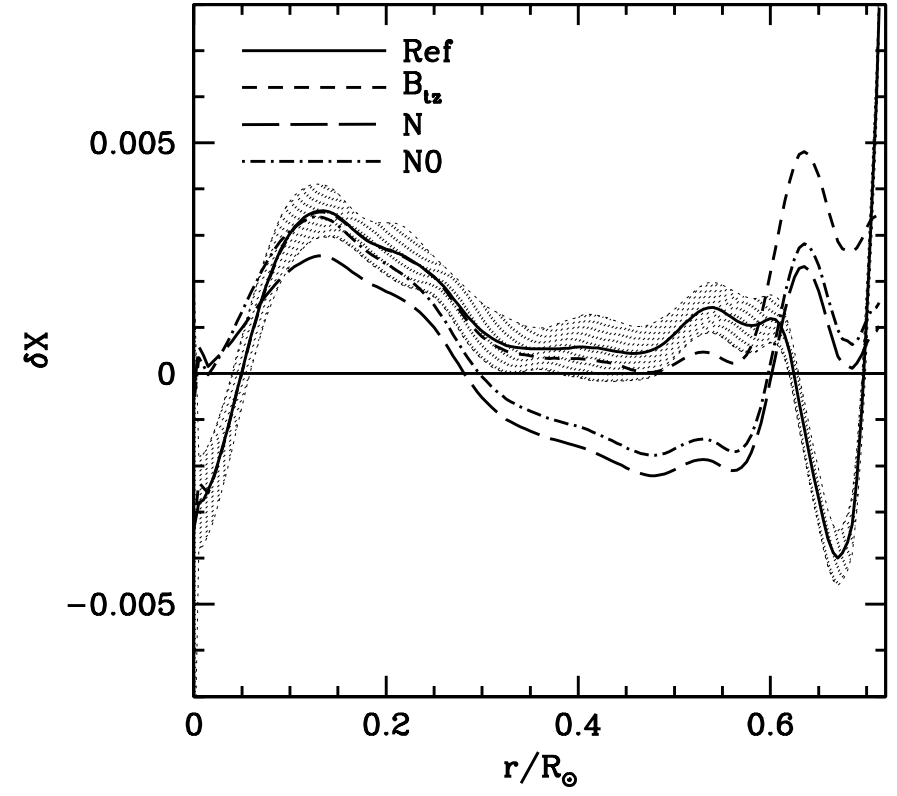

Fig. 8. The absolute difference in hydrogen abundance profile between the models and the Sun is shown as a function of fractional radius. Model ref including only microscopic diffusion and the mixed models $B_{t z} N 0$ and $N$ are represented respectively with solid, short-dashed, dash-dotted and long-dashed lines. Superimposed on model ref is the $1 \sigma$ error envelope coming from the helioseismic inversion.

closer to the inferred hydrogen abundance over the whole radiative interior (except for a small region between 0.35 and $0.5 R_{\odot}$ ) than either of the two other models shown. Further, Fig. 8 shows that the photospheric hydrogen content of model $N\left(X_{\mathrm{ph}}=0.7333\right.$ see Table 3$)$ and model $N 0$ are closer to the seismically inferred value of $0.732 \pm 0.001$ than both models $B_{t z}$ and ref. Thus the effect of varying the nuclear cross section $S_{11}$ is to change the hydrogen surface abundance in the model via the calibration procedure, the maximum amplitude of such modification being located in the central region, leading to an improvement of the agreement between the Sun and model $N$ by at least a factor 2 .

While determining the $X$ profile through inversions we also get an estimate of $p p$ reaction cross-section, $S_{11}$ that is required to match the observed solar luminosity. Table 3 lists the values obtained by assuming the $Z$ profile of each of the models considered in this study. It is clear that this estimate is not sensitive to other properties of the model, except for the treatment of the plasma screening. Beside model $N 0 W$, all other models yields a value $S_{11} \approx 4.06 \times 10^{-25} \mathrm{MeV}$ Barns, which is $1.5 \%$ higher than the value given by Adelberger et al. (1998). This is somewhat less than the value given by Antia \& Chitre (1999) or by Degl'Innocenti et al. (1998). This difference is due to different treatment of the plasma screening in calculating nuclear energy generation rates. If one uses weak, intermediate or strong screening for the solar plasma, the resulting increase in the $p p$ cross section $S_{11}$ found by seismic inversion will vary respectively between $0.5 \%$ and $4 \%$. Anyway all screening treatments seem to indicate a higher value of $S_{11}$ than currently calculated by nuclear physicists. However, it appears that with our intermediate screening (e.g., Mitler 1977), an increase of $S_{11}$ 
Table 3. Seismic inference on hydrogen abundance and $S_{11}$.

\begin{tabular}{cccc}
\hline \hline Models & $X_{\mathrm{ph}}$ & $X_{\text {inv }}$ & $S_{11}$ \\
\hline$R e f$ & 0.7392 & 0.7311 & 4.053 \\
$B_{t z}$ & 0.7304 & 0.7269 & 4.053 \\
$N 0$ & 0.7338 & 0.7322 & 4.066 \\
$N 02$ & 0.7339 & 0.7322 & 4.054 \\
$N 03$ & 0.7334 & 0.7323 & 4.085 \\
$N 0 W$ & 0.7337 & 0.7322 & 4.017 \\
$N$ & 0.7333 & 0.7323 & 4.067 \\
$N 1$ & 0.7334 & 0.7323 & 4.060 \\
$N 2$ & 0.7336 & 0.7323 & 4.054 \\
$N D$ & 0.7321 & 0.7327 & 4.057 \\
$N M$ & 0.7329 & 0.7324 & 4.055 \\
$N E$ & 0.7362 & 0.7317 & 4.048 \\
\hline
\end{tabular}

Note: $X_{\mathrm{ph}}$ and $X_{\text {inv }}$ correspond respectively to the photospheric hydrogen abundance achieved in the model and deduced by seismic inversion. The seismically deduced $p p$ reaction cross-section $S_{11}$ for each models is given in unit of $10^{-25} \mathrm{MeV}$ barns.

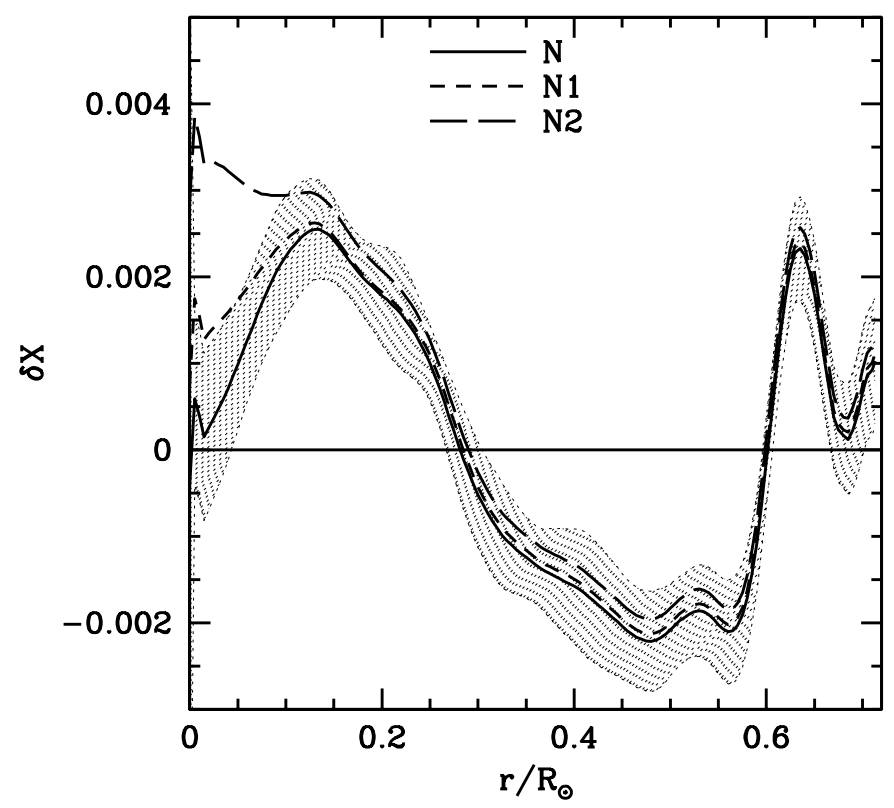

Fig. 9. The absolute difference in hydrogen abundance profile between solar models and the Sun as a function of fractional radius. The mixed models $N, N 1$ and $N 2$ are represented respectively with solid, shortdashed and long-dashed lines. Superimposed on model $N$ is the $1 \sigma$ error envelope from the helioseismic inversion.

by $3.5 \%$ pushes the value of the $p p$ cross section beyond what is required from seismic constraints, but nevertheless we keep these models as they amplify the effect of increasing $S_{11}$ and are in rather good agreement with the primary inversions of the sound speed and density.

Apart from $S_{11}$ Table 3 also gives the seismically inferred photospheric hydrogen abundance $\left(X_{\text {inv }}\right)$. Again this value is not sensitive to small differences in the $Z$ profile, but is mainly determined by the photospheric $Z$ value. Thus all models other than $B_{t z}$ give $X_{\mathrm{inv}} \approx 0.732$ which is only slightly less than the value in the corresponding solar model $\left(X_{\mathrm{ph}}\right)$. Further, this value will yield helium abundance, $Y \approx 0.25$, which is also

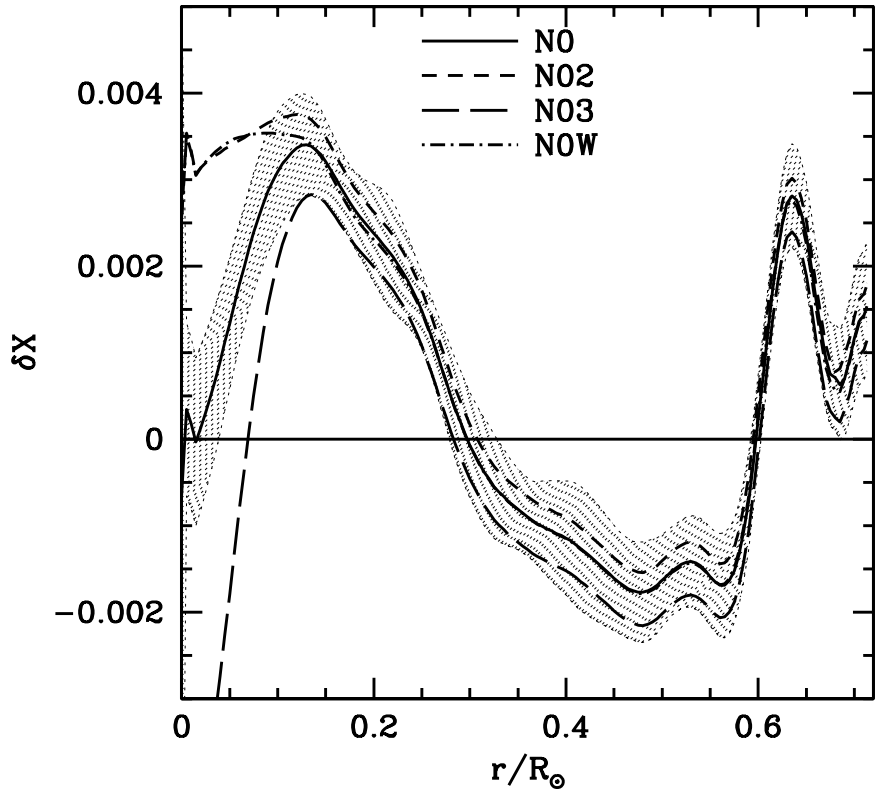

Fig. 10. The absolute difference in hydrogen abundance profile between solar models and the Sun. The mixed models N0, N02, N03 and $N 0 W$ are represented respectively with solid, short-dashed, longdashed and dot-dashed lines. Superimposed on model NO is the $1 \sigma$ error envelope from the helioseismic inversion.

close to the independently inferred value using seismic inversions in the convection zone (Basu 1998; DiMauro et al. 2002). Thus the photospheric helium abundances obtained using different techniques are consistent with each other.

We now consider the effect of a variation of the ${ }^{3} \mathrm{He}-{ }^{3} \mathrm{He}$ and ${ }^{3} \mathrm{He}-{ }^{4} \mathrm{He}$ reaction rates on the hydrogen abundance via their influence on the creation and destruction of helium in the solar core. In Fig. 9 we display the absolute difference $\delta X$ between the mixed models $N, N 1$ and $N 2$ and the Sun. As we previously did, we prefer to use the mixed model $N$ for comparison in this plot, since model $N$ is significantly closer to the seismic Sun than model ref is. The absolute differences $\delta X$ for the three models shown are all very close to zero. We find that as with the sound speed profile, variations of the nuclear reaction cross section $S_{34}$ modifies relatively more the hydrogen abundance profile than variations of $S_{33}$, but obviously less than modifications that variations of $S_{11}$ can produce. But for the quantity $\delta X$, model $N$ gives a better agreement with the Sun than models $N 1$ and $N 2$.

In Fig. 10 we display the difference in hydrogen abundance between the sequence of mixed models N0, N02, N03 and $N 0 W$ and the Sun. A first small difference with the previous figure is that an increase of $p p$ by $+2 \%$ does not reduce as much the disagreement with the inferred hydrogen abundance profiles than an increase by $3.5 \%$ does. As for model $N 2$, the hydrogen abundance profile of model N02 is only slightly affected in the inner central part. This seems to indicate that the variation of $-10 \%$ of $S_{34}$ is too large. Model N03, with opposite variations of $S_{33}$ and $S_{34}$ cross sections, is shifted downward by -0.0005 compared to model $N 0$ over most of the radiative zone and its core profile is way off. All these models confirm the feeling that the effects of the $S_{33}$ and $S_{34}$ cross 
section are mixed, in the sense that in some regions the agreement is improved while in other regions it becomes worse. Thus it is difficult to conclude if the variation of these cross sections is justified basing one's argument on seismic inversion of the hydrogen abundance.

For model $N 0 W$, computed with a weak nuclear screening instead of intermediate as in model N02, the result is a very small downward shift of $\delta X$ compared to model N02, but no significant improvement otherwise. It is thus unlikely that the screening effect will correct near the solar core the remaining discrepancy seen in the hydrogen abundance profiles obtained in our mixed models.

Finally in Fig. 11 we display the difference in hydrogen abundance between the solar models and the Sun for cases $N M$ and $N E$ that include different macroscopic parameters and case $N D$ with reduced microscopic diffusion along with case $N 2$. For model $N D$ the effect of the microscopic diffusion is subtle to be appreciated because it modifies non uniformly the hydrogen abundance. The model seems to possess the closest hydrogen surface abundance $X_{\mathrm{ph}}=0.7321$ relative to the inferred value $X_{\mathrm{inv}}=0.7327$, and the smallest composition gradient at the base of the convection zone, thus justifying the use of a smaller microscopic diffusion coefficient $D_{i}$. But the reduction seems overestimated because model $N D$ is the only solar model to exhibit a smaller hydrogen photospheric abundance $X_{\mathrm{ph}}$ than the seismically inferred one. Further, deeper down in the radiative interior the hydrogen abundance profile departs too much from the inferred profile. Therefore, as for the sound speed, a reduction of $D_{i}$ seems to be discarded by present helioseismic inversions.

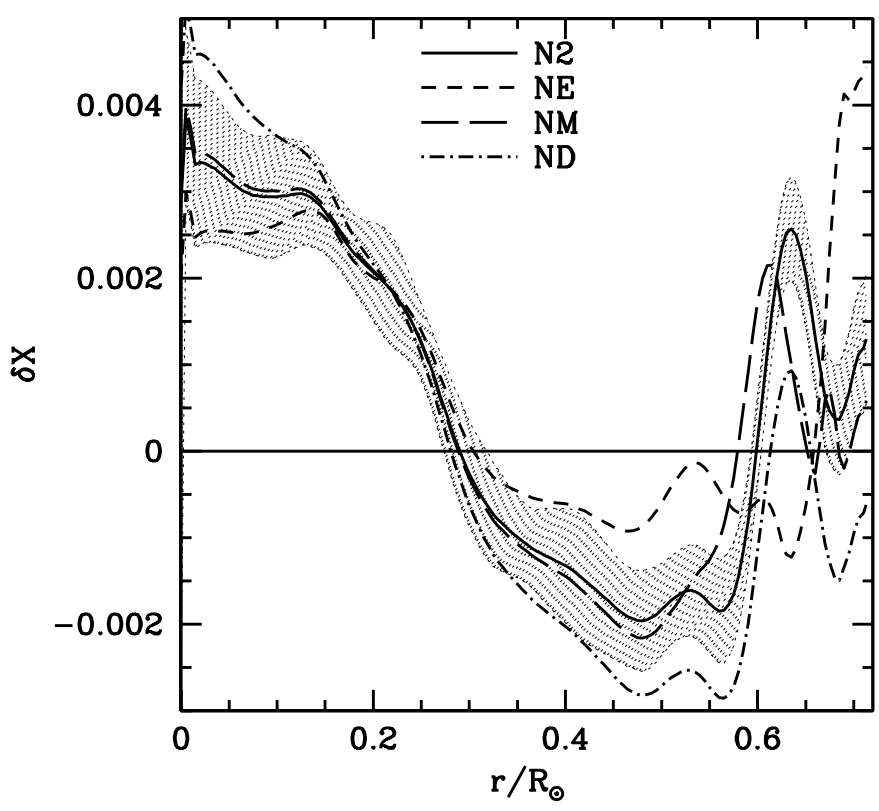

Fig. 11. The absolute difference in hydrogen abundance profile between solar models and the Sun as a function of fractional radius. The mixed models $N 2, N E, N M$ and $N D$ are represented respectively with solid, short-dashed, long-dashed, and dot-dashed lines. Superimposed on model $N 2$ is the $1 \sigma$ error envelope coming from the helioseismic inversion.
The effect of a broader mixing on $\delta X$ (i.e., model $N M$ ) is to reduce the composition gradient and to extend the mixed plateau, properties that seem to be in better agreement with the inferred $X$ profile. Moreover, $X_{\mathrm{ph}}$ is closer to $X_{\mathrm{inv}}$ in models $N M$ and $N D$ than for example model $N 2$. Deeper down the improvement is not as obvious and considering the fact that the extent of the tachocline mixing is certainly over estimated in this model, we can hardly conclude that it constitutes a better solution than, say, models $N$ or $N 2$. Model $N E$ is clearly worse in $X$ as well as in $\delta c / c$ and $\delta \rho / \rho$ compared to other mixed models. Whereas its profile deep in the radiative interior is in reasonable agreement with the seismically inferred one, while it is clearly not the case for the upper part $\left(r>0.6 R_{\odot}\right)$. The resulting composition gradient is too steep and the hydrogen abundance in the convective envelope too high.

To summarise our findings about the hydrogen abundance profile, it can be stated that "tachocline mixing" is very likely to occur in the Sun, and that an increase of $S_{11}$ is clearly favoured by current helioseismic data, with an amplitude of the order of a few \%. Less obvious are the effects of the two others dominant nuclear cross sections, i.e., $S_{33}$ and $S_{34}$, although they can lead to some improvement as well. On the other side, neither the microscopic diffusion nor the screening effect seem to cause significant changes. However, the screening prescription has been found to modify significantly the value of the cross section of the $p p$ nuclear reaction inferred by seismic inversion. Our last two models $N E$ and $N M$ including variations of the macroscopic parameters are not favoured by our study, because with model $N E$, the mixing is too shallow and does not vary with time and with model $N M$ the mixing is too broad. We thus find that the seismically inferred photospheric hydrogen abundance is $X_{\text {inv }}=0.732 \pm 0.001$, but the value change quite a bit depending on the value of heavy elements abundance assumed.

We would like now to briefly discuss the new photospheric composition obtained by our models at the solar age, with particular emphasis on light elements depletion. As already stressed, the mixed models exhibit a better overall chemical composition, say compared to models with only microscopic diffusion such as ref. One chemical element, namely the lithium, is crucial to assess the efficiency and time dependence of macroscopic mixing. With the presence of an effective macroscopic mixing at the base of the convection zone, all the models presented in this study are expected to deplete a fair amount of ${ }^{7} \mathrm{Li}$. All models, except $N E$, burn indeed a substantial quantity of ${ }^{7} \mathrm{Li}$ both in the PMS and in the main sequence phases, thus reaching a photospheric abundance at the solar age, $\mathrm{Li}_{\mathrm{s}}$, significantly smaller than the initial/meteoritic value, $\mathrm{Li}_{0}$, i.e., $\mathrm{Li}_{0} / \mathrm{Li}_{\mathrm{s}} \sim 130-180$, in reasonable agreement with the observations of Grevesse et al. (1996), $\mathrm{Li}_{0} / \mathrm{Li}_{\mathrm{s}} \sim 140$. Model $N E$ depletes a large amount of lithium as well $\left(\mathrm{Li}_{0} / \mathrm{Li}_{\mathrm{s}} \sim 100\right)$, but most of it $(\sim 90 \%)$ in the PMS phase, which is not realistic when compared with open cluster observations. This comes about because the macroscopic coefficient $D_{\mathrm{TE}}$ used in this model, following Elliott \& Gough treatment of the tachocline, does not include any time dependence. Brun et al. (1999) have demonstrated that a proper time dependence of $D_{\mathrm{T}}$ causes significant lithium burning along the main 
sequence as well. Another important constraint is provided by the beryllium abundance, which requires that the mixing must be shallow in order not to destroy this element by more than $10 \%$ (see Brun et al. 1999; Bell et al. 2001). With either of the two diffusion coefficients used in this study, $D_{\mathrm{T}}$ or $D_{\mathrm{TE}}$, we easily achieve this goal, i.e., all the models but one deplete ${ }^{9} \mathrm{Be}$ by less than $10 \%$. Model NM, which has been computed with the broadest tachocline mixing (i.e., $h=0.075 R_{\odot}$ ), leads to an underabundance of ${ }^{9} \mathrm{Be}$ of $20 \%$, thus confirming that in this model the mixing extends too deep inside the radiative zone. We interpret this result as an indication that the tachocline mixing can not be broader than $5 \%$ in solar radius. Thus using this upper limit for the tachocline extent, we are quite confident about the efficiency of our macroscopic time dependent coefficient $D_{\mathrm{T}}(r, t)$ to model the tachocline region and to lead not only to the proper photospheric composition at the solar age but also in the earlier phases. However, as already stated with model $B_{t z}$, with our prescription the lithium depletion is still too big in the PMS, even though it is reasonably distributed over the whole temporal evolution compared, say, to model $N E$.

\subsection{Temperature profile and neutrino production}

It has been known for more than thirty years that standard solar models and neutrino experiments on Earth disagree on the amount of neutrinos produced in the thermonuclear core of the Sun, the former predicting always a flux in excess (Bahcall 1989). A number of ingenious suggestions have been given to either explain the discrepancy from revised and "non-standard" solar models or by invoking neutrino flavour oscillations from the electron neutrino $v_{\mathrm{e}}$, generated in the $p p$ chains and $\mathrm{CNO}$ cycles, to its siblings the muon $v_{\mu}$ and tau $v_{\tau}$ neutrinos or to the so-called "sterile neutrino" (Haxton 1995; Bahcall et al. 1998b). Here we intend to use the seismic diagnosis to constrain as much as possible the theoretical neutrino flux, which is very sensitive to the central temperature.

Figure 12 represents the relative temperature difference between solar models ref, $B_{t z}, N, N 0, N 02$ and N03 and the Sun. The overall agreement of the six models is quite satisfactory, with model ref being the least accurate in the tachocline region as expected.

We can indeed notice that the introduction of a macroscopic mixing in the tachocline improves the profile of all the models by at least a factor 2 above $r=0.6 R_{\odot}$, over model ref, thus confirming the importance of taking into account the mixing present in this transition region. In the bulk of the radiative zone, some improvement comes from the increase of the $p p$ cross section and results in a flattening of $\delta T / T$ and a slightly closer agreement with the Sun. For the very central part, where the neutrinos are produced, the secondary seismic inversion of the temperature is less accurate with an error bar of $\pm 2 \times 10^{-3}$, and thus does not constrain the solar models as much. Models ref and $B_{t z}$ are surprisingly good there. Model $N$ is quite satisfying except in this very central part. This can be interpreted as an excessive increase of the $p p$ cross section. Models $N 0$ and $N 03$ seem to be our best models in the range $[0,0.7] R_{\odot}$. While this could have been expected from model

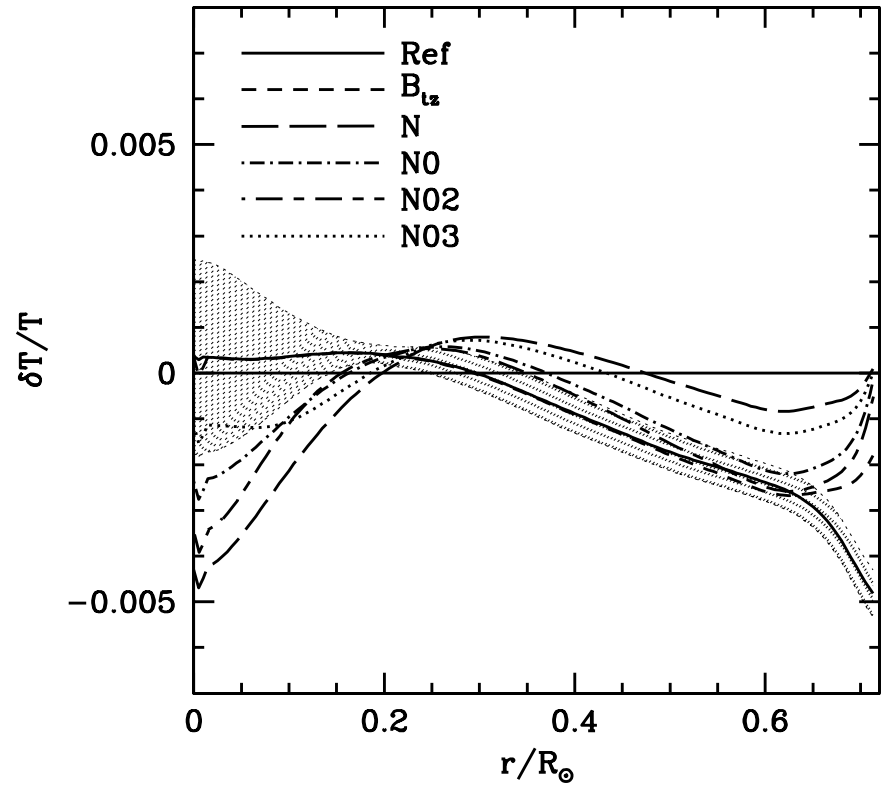

Fig. 12. The relative temperature difference between solar models ref, $B_{t z}, N, N 0, N 02$ and $N 03$ and the Sun. Note the somewhat larger uncertainties for this variable in the neutrino production region $(r<$ $0.3 R_{\odot}$ ) compared for example to the sound speed ones.

$N 0$, based on the inversion of the sound speed and density profiles (Sect. 3.2), it was not so for model $\mathrm{N03}$ that has been computed with opposite variation of the nuclear cross sections $S_{33}$ and $S_{34}$. Effectively, it seems that for the temperature profile, the decrease of $S_{34}$ by $10 \%$, as in model $N 02$, does not lead to any progress in the central part while an increase does. This conclusion is at odd with what we learnt from the sound speed inversion. This could mean that either the secondary inversion of the temperature is not as reliable as the sound speed (that is in part true but unsatisfactory) or that the temperature varies differently with modifications of the main physical ingredients than the sound speed does, due for example to a compensatory change of the central composition. So, we have to be cautious in our conclusions regarding the very central part since this is the region where the inversions are the least reliable. Nevertheless, we still consider that for the temperature profile models ref, $B_{t z}, N 0, N 03$ and more marginally, model $N$, all represent seismically acceptable solutions of the solar core. As a result, what one can expect to be the impact on the neutrino fluxes of such diverse temperature profiles?

To answer that question we have summarised in Table 4 the neutrinos fluxes of the most significant models. By comparing for example models ref and $N 2$, we find that for the latter the Gallium flux is reduced by 8 SNU, the Chlorine by 1.34 SNU and the ${ }^{8} \mathrm{~B}$ by $10^{6} \mathrm{~cm}^{-2} \mathrm{~s}^{-1}$ down to $3.93 \times 10^{6}$ $\mathrm{cm}^{-2} \mathrm{~s}^{-1}$. Such theoretical fluxes are still too high compared to the neutrino experiment on Earth (cf., Table 1), if no other modifications are introduced either in the model or in the quantum properties of the neutrinos. For example, the production of the ${ }^{8} \mathrm{~B}$ neutrinos is directly sensitive to the $p$ ${ }^{7} \mathrm{Be}$ nuclear cross section. The value used in this study is $S_{17}(0)=19.1_{-2}^{+4} \mathrm{eV}$ barns (Adelberger et al. 1998), and corresponds to an intermediate value compared to the recent 
Table 4. Neutrino fluxes at Earth.

\begin{tabular}{|c|ccc|}
\hline \hline & \multicolumn{3}{|c|}{ Detector } \\
Models & ${ }^{71} \mathrm{Ga}$ & ${ }^{37} \mathrm{Cl}$ & Water \\
\hline \hline$R e f$ & 127.1 & 7.04 & 4.99 \\
$B_{t z}$ & 127.1 & 7.04 & 4.99 \\
\hline \hline$N 0$ & 123.7 & 6.41 & 4.48 \\
$N 03$ & 128.2 & 7.08 & 4.99 \\
$N 0 W$ & 121.4 & 6.10 & 4.25 \\
$N$ & 122.5 & 6.18 & 4.29 \\
$N 1$ & 121.3 & 6.0 & 4.16 \\
$N 2$ & 119.3 & 5.7 & 3.93 \\
$N D$ & 118.7 & 5.59 & 3.85 \\
\hline
\end{tabular}

Note: The ${ }^{71} \mathrm{Ga},{ }^{37} \mathrm{Cl}$ and Water columns correspond respectively to the predicted solar neutrinos fluxes for the gallium, chlorine and water experiments (cf., Table 1). The gallium and chlorine neutrino fluxes are given in SNU whereas the water (SNO/SuperKamiokande) ones are in $10^{6} \mathrm{~cm}^{-2} \mathrm{~s}^{-1}$.

estimate of Davids et al. (2001) (i.e., $S_{17}(0)=17.8_{-1.2}^{+1.4} \mathrm{eV}$ barns) or of Junghans et al. (2002) (i.e., $S_{17}(0)=22.3 \pm 0.7 \pm$ $0.5 \mathrm{eV}$ barns). Thus we can conservatively consider that the error bar in $S_{17}$ nuclear cross section is at least of the order of $\pm 10 \%$. Such an uncertainty, results in an increase or decrease of our ${ }^{8} \mathrm{~B}$ flux by $\pm 10 \%$ as well.

By taking into account most of the uncertainties present in a solar model to calculate the neutrino fluxes, such as the nuclear reactions cross sections, the screening, the heavy elements abundance, the absorption cross sections for gallium and chlorine experiments, the amount of microscopic and macroscopic diffusion, etc. (see Turck-Chièze et al. 2001), we end up with the following fluxes (errors have been obtained by quadratic sum of the individual contributions assumed to be independent):

${ }^{71} \mathrm{Ga}=123.7 \pm 8.7 \mathrm{SNU}$,

${ }^{37} \mathrm{Cl}=6.41 \pm 0.86 \mathrm{SNU}$ and

Water $=(4.48 \pm 0.71) \times 10^{6} \mathrm{~cm}^{-2} \mathrm{~s}^{-1}$.

These values are a bit lower than Bahcall et al. (2001) and Turck-Chièze et al. (2001), but remain within the $1 \sigma$ error range. However, they are still significantly larger than the observations, unless one invokes non standard neutrino properties. Such evidence for neutrino flavour oscillation have been recently given by Ahmad et al. (2001) based on the careful study of the high energy neutrino fluxes detected by the SNO detector and by the SuperKamiokande experiments (Fukuda et al. 1998). Since then SNO has also measured the ${ }^{8} \mathrm{~B}$ neutrino flux using the neutral current channel, which is equally sensitive to all neutrino flavours (Ahmad et al. 2002). The resulting ${ }^{8} \mathrm{~B}$ flux, is found to be $(5.09 \pm 0.62) \times 10^{6} \mathrm{~cm}^{-2} \mathrm{~s}^{-1}$, which is within the error bars of the current solar neutrino prediction. Our helioseismic study seems to favour more the lower range of the detection than the upper range.

\section{Discussion and conclusions}

In this work our goal was to assess the effect of uncertainties in nuclear reaction rates, atomic data and diffusion coefficients on solar models, which we have compared with the results of helioseismic inversions. We have concluded in Sect. 3 that a variation of the nuclear reaction rates can have a significant impact on the solar structure and that current modelling coupled with seismic data favours some change of the accepted central value of three important nuclear reaction rates, $p p,{ }^{3} \mathrm{He}-{ }^{3} \mathrm{He}$ and ${ }^{3} \mathrm{He}-{ }^{4} \mathrm{He}$. While the $p p$ reaction rates needs to be increased by about $1.5 \%$ over the currently accepted value from Adelberger et al. (1998), the constraints on other reaction rates are less clear. Even the increase of $p p$ rate involved in this study is less than that inferred by Antia \& Chitre (1999) and Degl'Innocenti et al. (1998) and the difference can be attributed to different treatment of nuclear energy generation in CESAM as compared to the version of Bahcall's energy routine used in earlier estimates. More precisely, most of the discrepancy comes from the different screening prescriptions used. In the previous study of Antia \& Chitre (1999), the screening formulation of Graboske et al. (1973) was assumed as opposed to Salpeter (1954) or Mitler (1977) in this new study. We refer to Dzitko et al. (1995) and Wilets et al. (2000) for a detailed comparison of several weak, intermediate and strong, screening prescriptions.

Our work confirms the result of Brun et al. (1999) that the implementation of macroscopic mixing in the tachocline improves the agreement between solar models and seismic Sun (Sect. 3.2). In particular, direct comparison of hydrogen abundance profiles between our models and the Sun as inferred from seismic inversions has demonstrated that the models with tachocline mixing are in much better agreement with the Sun in tachocline region. Comparison of hydrogen abundance profile in solar models with inferred profiles show that there is still a discrepancy of about 0.003 (Sect. 3.3). The largest discrepancies occur in the region close to the tachocline and at about $r=0.2 R_{\odot}$, where the sound speed also shows maximum discrepancy. Thus it appears that there is still some scope for improving the formulation for calculating the mixing in tachocline region. We were not able to achieve any significant improvement compared to the results of Brun et al. (1999) by adjusting the parameters of the tachocline model. With some modifications the agreement improves in the tachocline region, like in model $N M$, but it tends to worsen in other places. We have found as well that based on the ${ }^{9} \mathrm{Be}$ photospheric depletion, the maximum extent of the mixing in the tachocline is $5 \%$ of solar radius. It is quite possible that a major part of the remaining discrepancies (about $0.1 \%$ in sound speed, $1 \%$ in density and 0.003 in $X$ ) in our improved models may be due to uncertainties in input physics, like the opacities or equation of state.

Comparing the surface hydrogen abundance in solar models and those obtained by inversions (Table 3) with the abundance inferred directly from seismic data (Basu 1998), we again find a difference of 0.001 . This suggests that discrepancies of this order are present in the input physics which is used in inferring these independent measurements of surface 
hydrogen abundance. The helium abundance drawn from the structure of the ionisation zones is particularly sensitive to the equation of state (Basu \& Antia 1995), while its abundance inferred from the solar models depends on the whole input physics used in constructing those. Similarly, the seismically inferred hydrogen abundance profile is sensitive to opacity as well as to the $Z$ profile used in inversion. Thus the discrepancy between these three independent estimates of surface hydrogen abundance probably gives an estimate of errors in input physics. The difference between solar models with tachocline mixing and seismically inferred profiles is of the same order and could be due also to remaining uncertainties in input physics.

As seen in Fig. 1 the evolutionary solar models show a significant departure from seismic inferences inside the convection zone. We find that scaling the radius of these models by 1.0003 before taking the differences with inverted profile removes most of the discrepancy in the convection zone. We believe that this is due to uncertainties in the treatment of surface layers. In the solar models the surface is defined by the layer where the temperature equals effective temperature and it is quite possible that because of uncertainties in surface layers the location of this point has an error of about $200 \mathrm{~km}$ in our evolutionary models. We would like to point out that adjusting the solar radius in the model by $200 \mathrm{~km}$ does not remove this discrepancy, since even in the new model the position of the surface has the same uncertainty. Thus this scaling of radius has no relation to uncertainty in solar radius itself.

The calculated neutrino fluxes in solar models with tachocline mixing are found to be somewhat lower than those in standard solar model of Bahcall et al. (2001), but within the $1 \sigma$ error limits (Sect. 3.4). The main reason for reduction is the increase in $p p$ reaction rate, which reduces the central temperature required to generate the solar luminosity. Recently, using both the charged and neutral current channels, SNO has measured the total ${ }^{8} \mathrm{~B}$ neutrino flux of $5.09 \times 10^{6} \mathrm{~cm}^{-2} \mathrm{~s}^{-1}$ (Ahmad et al. 2002). This is somewhat larger than the value we find in our models. In the modified models $N 1, N 2$ the neutrino fluxes are much lower, because we have chosen to modify the nuclear reaction rates to reduce these fluxes. If instead as with model N03 we had modified $S_{33}$ and $S_{34}$ in opposite directions the neutrino fluxes would have increased and it would be possible to get values close to that inferred by Ahmad et al. (2002). Also our increase of $p p$ reaction rate by $3.5 \%$ in some of these models is probably an overestimate, since seismic models with same input physics appear to need an increase by only $1.5 \%$ to produce the required solar luminosity. When almost all the sources of uncertainties are taken into account in evaluating the theoretical neutrino fluxes, our result is within $1 \sigma$ of the other published theoretical neutrino fluxes and the recent SNO compilation. It will be interesting in the near future, when the SNO collaboration results will have been integrated over a longer time to see if the helioseismic tool will be able to constrain even more efficiently the main nuclear cross sections, via a careful analysis of the neutrino spectrum, and also delineate the parameter space for mass-squared difference-mixing angle plane.
Acknowledgements. We thank the anonymous referee for constructive comments and suggestions which have led to a considerable improvement in the final version of the paper. ASB would like to thank S. Turck-Chièze for useful discussions and the Service d'Astrophysique, for access to their computers. This work was partly supported by NASA through grants NAG5-2256 and NAG58133. SMC is grateful to DAE-BRNS for support under the Senior Scientist Scheme.

\section{References}

Adelberger, E. G., Austin, S. M., Bahcall, J. N., et al. 1998, Rev. Mod. Phys., 70, 1265

Ahmad, Q. R., Allen, R. C., Andersen, T. C., et al. 2001, Phys. Rev. Lett., 87, 071301 (SNO collaboration)

Ahmad, Q. R., Allen, R. C., Andersen, T. C., et al. 2002, Phys. Rev. Lett., 89, 011301 (SNO collaboration)

Angulo, C., Arnould, M., Rayet, M., \& the NACRE collaboration 1999, Nucl. Phys. A, 656, 3

Antia, H. M. 1996, A\&A, 307, 609

Antia, H. M., \& Chitre, S. M. 1998, A\&A, 339, 239

Antia, H. M., \& Chitre, S. M. 1999, A\&A, 347, 1000

Antia, H. M., Basu, S., \& Chitre, S. M. 1998, MNRAS, 298, 543

Bahcall, J. N. 1989, Neutrino Astrophysics (Cambridge University Press)

Bahcall, J. N., Basu, S., \& Pinsonneault, M. H. 1998a, Phys. Lett. B, 433,1

Bahcall, J. N., Krastev, P. I., \& Smirnov, A. Yu. 1998b, Phys. Rev. D, 58, 096016

Bahcall, J. N., Pinsonneault, M. H., \& Basu, S. 2001, ApJ, 555, 990

Balachandran, S., \& Bell, R. A. 1998, Nature, 392, 791

Barnes, G., Charbonneau, P., \& MacGregor, K. B. 1999, ApJ, 511, 466

Basu, S. 1998, MNRAS, 298, 719

Basu, S., \& Antia, H. M. 1994, MNRAS, 269, 1137

Basu, S., \& Antia, H. M. 1995, MNRAS, 276, 1402

Basu, S., Turck-Chièze, S., Berthomieu, G., et al. 2000, ApJ, 535, 1078

Bell, R. A., Balachandran, S., \& Bautista, M. 2001, ApJ, 546, L65

Brun, A. S., Turck-Chièze, S., \& Morel, P. 1998, ApJ, 506, 913

Brun, A. S., Turck-Chièze, S., \& Zahn, J.-P. 1999, ApJ, 525, 1032

Canuto, V. M., \& Mazzitelli, I. 1991, ApJ, 370, 295

Cayrel, R. 1998, Space Sci. Rev., 84, 145

Christensen-Dalsgaard, J., Berthomieu, G. 1991, in Solar interior and atmosphere, ed. A. N. Cox, W. C. Livingston \& M. Matthews (University of Arizona Press), 401

Christensen-Dalsgaard, J., Gough, D. O., \& Thompson, M. J. 1991, ApJ, 378, 413

Christensen-Dalsgaard, J., Proffitt, C. R., \& Thompson, M. J. 1993, ApJ, 403, L75

Christensen-Dalsgaard, J., Däppen, W., et al. 1996, Science, 272, 1286

Clayton, D. D. 1968, Principles of Stellar Evolution and Nucleosynthesis (University of Chicago Press)

Corbard, T., Blanc-Féraud, L., Berthomieu, G., \& Provost, J. 1999, A\&A, 344, 696

Davids, B., Austin, S. M., Bazin, D., et al. 2001, Phys. Rev. Lett., 86, 2750

Degl'Innocenti, S., Fiorentini, G., \& Ricci, B. 1998, Phys. Lett. B, 416, 365

Di Mauro, M. P., Christensen-Dalsgaard, J., Rabello-Soares, M. C., \& Basu, S. 2002, A\&A, 384, 666

Dzitko, H., Turck-Chièze, S., Delbourgo-Salvador, P., \& Lagrange, G. 1995, ApJ, 447, 428

Elliott, J. R., \& Gough, D. O. 1999, ApJ, 516, 475 
Fröhlich, C., Andersen, B. N., Appourchaux, T., et al. 1997, Sol. Phys., 170,1

Fukuda, Y., Hayakawa, T., Ichihara, E., et al. 1998, Phys. Rev. Lett., 81, 1158 (SuperKamiokande Collaboration)

Gabriel, A. H., Charra, J., Grec, G., et al. 1997, Sol. Phys., 175(2), 207

Gautier, D., \& Morel, P. 1997, A\&A, 323, L9

Gough, D. O., Kosovichev, A. G., Toomre, J., et al. 1996, Science, 272, 1296

Graboske, H. C., DeWitt, H. E., Grossman, A. S., \& Cooper, M. S. 1973, ApJ, 181, 457

Grevesse, N., Noels, A., \& Sauval, A. J. 1996, in Cosmic Abundances, ed. S. S. Holt, \& G. Sonneborn (San Francisco: ASP), 117

Haxton, W. C. 1995, ARA\&A, 33, 459

Haxton, W. C. 1997 [nucl-th/9704052]

Iglesias, C., \& Rogers, F. J. 1996, ApJ, 464, 943

Junghans, A. R., Mohrmann, E. C., Snover, K. A., et al. 2002 [nucl-ex/0111014]

Kippenhahn, R., \& Weigert, A. 1994, Stellar Structure and Evolution (Springer-Verlag, Berlin)

Kurucz, R. L., Stellar Atmospheres: Beyond classical models, ed. L. Crivellari, I. Hubeny, \& D. G. Hummer, NATO ASI Ser. (Kluwer, Dordrecht), 1991

Michaud, G., \& Proffitt, C. R. 1993, Inside the stars IAU 137, ed. W. W. Weiss, \& A. Baglin (San Francisco: ASP), 246

Mitler, H. E. 1977, ApJ, 212, 513

Montalban, J., \& Schatzman, E. 1996, A\&A, 305, 513
Morel, P. 1997, A\&AS, 124, 597

Morel, P., Provost, J., \& Berthomieu, G. 1997, A\&A, 327, 349

Morel, P., Pichon, B., Provost, J., \& Berthomieu, G. 1999, A\&A, 350, 275

Piau, L., \& Turck-Chièze, S. 2002, ApJ, 566, 419

Richard, O., Vauclair, S., Charbonnel, C., \& Dziembowski, W. A. 1996, A\&A, 312, 1000

Rogers, F. J., \& Iglesias, C. 1998, Space Sci. Rev., 85, 61

Salpeter, E. E. 1954, Aust. J. Phys., 7, 373

Schlattl, H., Bonanno, A., \& Paternó, L. 1999, Phys. Rev. D, 60, 113002

Schou, J., Antia, H. M., Basu, S., et al. 1998, ApJ, 505, 390

Skumanich, A. 1972, ApJ, 171, 565

Spiegel, E. A., \& Zahn, J.-P. 1992, A\&A, 265, 106

Thompson, M. J., Toomre, J., Anderson, E. R., et al. 1996, Science, 272,1300

Turck-Chièze, S. 1998, Space Sci. Rev., 85, 125

Turck-Chièze, S., Couvidat, S., Kosovochev, A. G., et al. (GOLF team E) 2001, ApJ, 555, L69

Turcotte, S., Richer, J., Michaud, G., Iglesias, C. A., \& Rogers, F. J. 1998, ApJ, 504, 539

Vernazza, J. E., Avrett, E. H., \& Loeser, R. 1981, ApJS, 45, 635

Weiss, A., Flaskamp, M., \& Tsytovich, V. N. 2001, A\&A, 371, 1123

Wilets, L., Giraud, B. G., Watrous, M. J., \& Rehr, J. J. 2000, ApJ, 530, 504

Zahn, J.-P. 1998, Space Sci. Rev., 85, 79 